\title{
Global Problems on Nash Functions
}

\author{
Michel Coste ${ }^{*}$, Jesús M. RuIz ${ }^{*} \dagger$ \\ and Masahiro SHIOTA
IRMAR (UMR CNRS 6625)
Université de Rennes I,
Campus de Beaulieu
35042 Rennes Cedex, France \\ michel.coste@univ-rennes1.fr
Departamento de Geometría y Topología, Facultad de Matemáticas,
Universidad Complutense 28040 Madrid, Spain
jesusr@mat.ucm.es \\ Graduate School of Polymathematics \\ Nagoya University, Chikusa \\ Nagoya, 464-01, Japan \\ shiota@math.nagoya-u.ac.jp
}

Recibido: 10 de Octubre de 2002

Aceptado: 2 de Junio de 2003

\begin{abstract}
This is a survey on the history of and the solutions to the basic global problems on Nash functions, which have been only recently solved, namely: separation, extension, global equations, Artin-Mazur description and idempotency, also noetherianness. We discuss all of them in the various possible contexts, from manifolds over the reals to real spectra of arbitrary commutative rings.
\end{abstract}

Key words: Nash function, étale morphism, cohomology, Cartan's theorems A and B, finite sheaf, semialgebraic topology, approximation theorem, real spectrum.

2000 Mathematics Subject Classification: 14P20, 32C07.

${ }^{*}$ Member of the European Research Training Network RAAG (Contract No. HPRN-CT-200100271).

†Partially supported by BFM2002-04797.

Rev. Mat. Complut. 


\section{Introduction}

Nash functions appeared for the first time at the early fifties, in a seminal paper by John Nash. Soon other mathematicians were interested on them, and some relevant applications found, but also soon their bad cohomological behaviour was realized. This put temporarily an end to a possible systematic development of the theory of Nash functions. Since that moment many specialists have devoted time and efforts to understand the nature of these functions, but only after fifty years we start to understand what is behind their failures, and how the problems generated by those failures should be settled. During this fifty years process the notion of Nash function has evolved in progressively more general settings, with an appealing feedback from concrete to abstract and viceversa. Moreover, a maze of surprising links have been revealed among all the questions involved. These are depicted in the Nash Labyrinth shown beside, where each box contains a solved problem, each arrow is an implication, and the doubled boxes are the sources of the flow of arguments. We will try to explain the problems and their solutions in the following sections:

1. Brief history of the topic

2. The notion of Nash function

3. Global properties and cohomological failures

4. Formulation of the problems

5. Solutions in the compact case

6. Solutions in the non-compact case

7. Nash functions over arbitrary real closed fields 


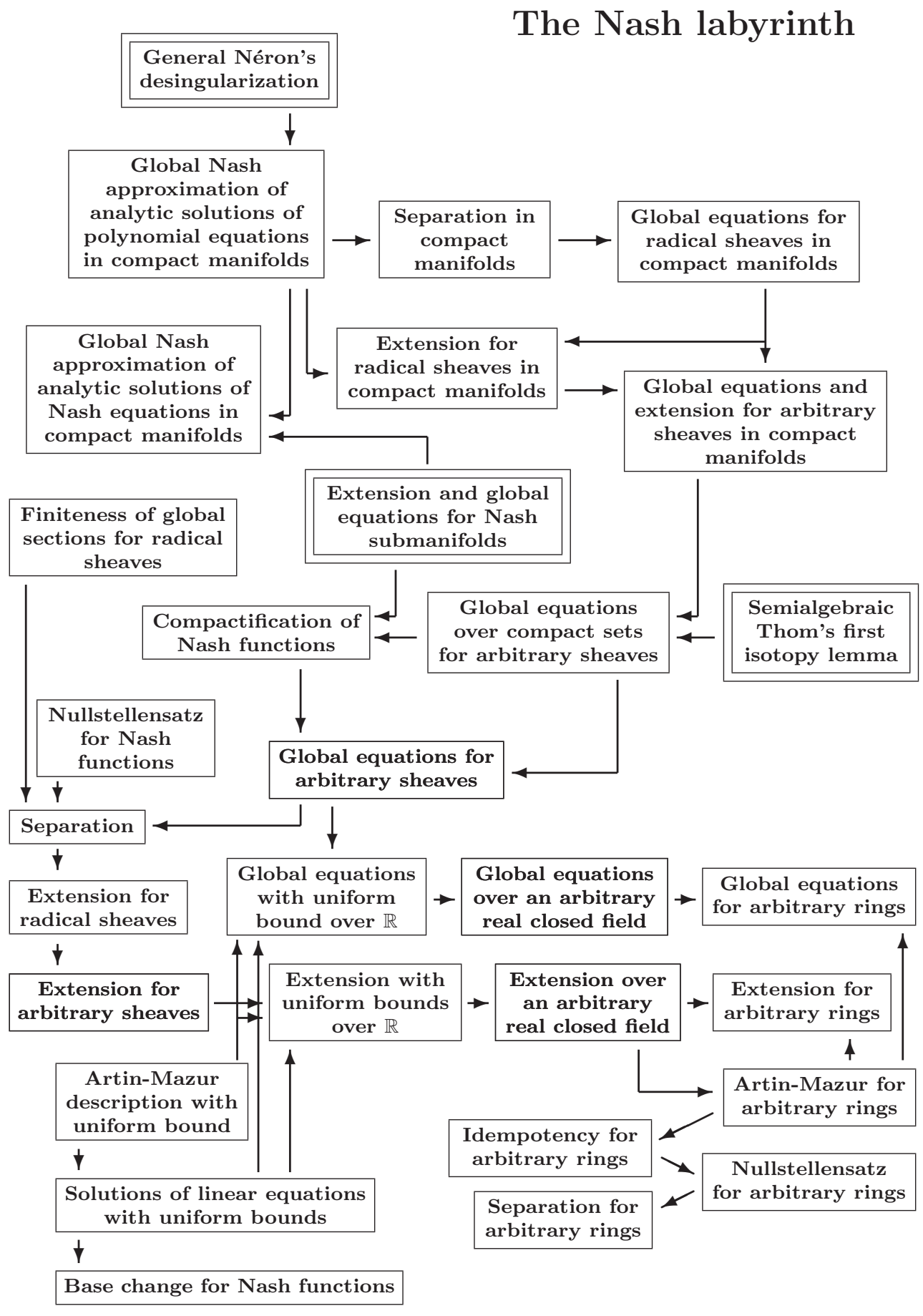




\section{Brief history of the topic}

In 1952 Nash published a paper [Na], whose final goal was to equip any given analytic manifold with a real algebraic structure. He did it completely in the compact case, by prescribing a smaller ring of analytic functions: one containing the coordinates of some embedding, with transcendence degree over the reals equal to the dimension of the manifold, and maximal for these properties. The proofs in the paper are a great example of the differential topology methods we are familiar with today: tubular neighborhoods and approximation. Nash added a careful analysis of the analytic functions which are roots of polynomials, now known after him Nash functions. Today we formulate Nash's result as that every compact smooth manifold is diffeomorphic to a connected component of a real algebraic set. In fact, we can get rid of the extra components, and the manifold is diffeomorphic to a non singular real algebraic set. This important improvement is due to Tognoli ([To]), but came 20 years later and only after Milnor's algebraic representation of Thom's cobordism classes (more differential topology).

Upon the publication of such a pioneering work, Nash functions were used with some success in several applications. The most relevant might be the one by Artin and Mazur, who in [ArMz] showed that every differentiable diffeomorphism may be approximated by others whose (finite) number of isolated periodic points grow at most exponentially with respect to the period; from this they deduced some other facts concerning numerically stable functions and structurally stable diffeomorphisms. Artin-Mazur's idea was to complement Nash methods with others typical of algebraic geometry, particularly the use of étale morphisms. Étaleness says algebraically when a polynomial map between algebraic sets is a local diffeomorphism. This is, of course, related to the existence of a local inversion theorem (or, equivalently, an implicit function theorem) in algebraic geometry. The notion of "local" which is used here refers to the étale topology, which is a Grothendieck topology and not a topology in the usual sense. However, an algebraic local inversion theorem can be obtained for the euclidean topology, using Nash functions. Actually, Nash functions form the smallest class of analytic functions which include all solutions of non-degenerate polynomial systems of equations.

At the same time Artin and Mazur presented their view of Nash functions, there was some systematic work on their local properties (Artin again, always interested on algebraicity matters, Lafon [Lf], Lazzeri and Tognoli [LaTo]). However, this start up was soon stopped when Hubbard $([\mathrm{Hb}])$ published an example in the real line of a Nash cocycle which is not a coboundary: what to do then with a class of functions whose cohomology is not even trivial on the real line?, how to deal with global questions?, what is the use of sheaf theory there? As partitions of unity are not Nash (the identity principle excludes bump functions), they are not available to glue local data. But then, cohomology is of no help either. Another example of a different nature, but also discouraging: when one sees the circle as a quotient $C=\mathbb{R} / \mathbb{Z}$, Nash functions on $C$ are periodic Nash functions on the line, hence all are constant. 
All these considerations put Nash functions aside, as long as a global problem was involved. Neither the redescovering of Real Algebraic Geometry at the end of the 70's, nor the renewed appeal of Nash's original ideas, made less suspicious the specialists, despite some important papers on global matters (see [Ef1],[Ef2], for instance). They were convinced that before solving in some satisfactory way their cohomological difficulties, Nash functions would be truly defective. Some tried to attack the problem, but there were few substantial advances. In fact, only in the 80's there was a serious attempt by Efroymson ([Ef3],[Ef4]) to bring more attention to the difficulties involved and find some, although partial, very interesting solutions. One must stress here how influential Efroymson's work was: specialists keep browsing through his (intrincate) writings to complete them or get some new insight (see $[\mathrm{Pe}],[\mathrm{CoDp}]$ ). After Efroymson, the main contributions on Nash functions were made by Shiota ([Sh1],[Sh2]). This attracted some other researchers (Beretta, Tancredi, Tognoli, see $[\mathrm{BeTo}]$ and $[\mathrm{TaTo} 1])$. Then, we wait ten years to really put everything in order and produce complete solutions. This final part of the story started with [RzSh], where the methods of Commutative Algebra were fetched to the stage, and finally the series [CoRzSh1], [CoRzSh2], [Qz2], [Qz3], [CoSh2], [CoRzSh3], which appeared from 1995 to 2001 and clarified everything.

\section{The notion of Nash function}

A Nash function on a connected open subset $U$ of $\mathbb{R}^{n}$ is a smooth function $f: U \rightarrow \mathbb{R}$ for which there is a polynomial $P(\mathrm{x}, \mathrm{t})$ in $n+1$ indeterminates $(\mathrm{x}, \mathrm{t})=\left(\mathrm{x}_{1}, \ldots, \mathrm{x}_{n}, \mathrm{t}\right)$ such that $P(x, f(x))=0$ for every $x \in U$. Nash functions have several important finiteness properties, easy to describe for one variable: a Nash function in one variable has finitely many zeros. Indeed, if $f(x)=0$, then $P(x, 0)=0$, and $x$ is a root of the polynomial $P(\mathrm{x}, 0)$. There are only finitely many such roots, except in case $P(\mathrm{x}, 0) \equiv 0$, but then $P(\mathrm{x}, \mathrm{t})=\mathrm{t} Q(\mathrm{x}, \mathrm{t})$, so that $f(x) Q(x, f(x))=0$ in $U$. Hence, either $f \equiv 0$, or $Q(x, f(x))=0$ in $U$. Choosing $P$ of minimal degree, we get a contradiction. In particular, we see why there are not non-constant periodic functions. This finiteness quality is an old pet of Real Geometry, and is perfectly expressed by the modern notion of o-minimal structure (see [vdD]); the book [Sh3] goes along the same ideas.

The essential contribution in $[\mathrm{ArMz}]$ to the understanding of Nash functions is that a function $f: U \rightarrow \mathbb{R}$ on a connected open subset $U$ of $\mathbb{R}^{n}$ is Nash if and only if there exist:

(i) A non singular algebraic set $V \subset \mathbb{R}^{n+k}$ of dimension $n$ such that the projection $\pi: V \rightarrow \mathbb{R}^{n}$ is étale (i. e. a local diffeomorphism),

(ii) A continuous (hence, analytic) section $\sigma: U \rightarrow V$ of the projection $\pi$,

(iii) A polynomial function $g: V \rightarrow \mathbb{R}$, 
such that $f=g \circ \sigma$. This Artin-Mazur description is a useful tool to retreat from Nash functions to polynomial ones.

Nash functions are analytic, and form a sheaf $\mathcal{N}_{\mathbb{R}^{n}}$ on $\mathbb{R}^{n}$. The stalk $\mathcal{N}_{\mathbb{R}^{n}, a}$ of that sheaf at a point $a \in \mathbb{R}^{n}$ consists of all Nash function germs at that point, and through Taylor expansion, those germs can be identified with the algebraic power series, that is, the real power series which are algebraic over the polynomials. Thus, the ring $\mathcal{N}_{\mathbb{R}^{n}, a}$ is the henselization of the local ring at $a$ of regular functions and has excellent algebraic properties: jacobian criteria, inverse and implicit functions theorem, Weierstrass preparation and division theorems, Artin's approximation theorem (see [BoCoRo]). These are the essential tools for the local study of zero sets of Nash functions, called Nash sets. A Nash set which is a smooth manifold is simply called a Nash manifold. These Nash sets, even with singularities, can be stratified in a controlled way, as finite disjoint unions of Nash manifolds, called strata, with good behaviour at the boundaries. Nash manifolds can be always compactified, as interiors of compact Nash manifolds with boundary. All of this seems quite natural, although there are some surprising technicalities (like uniqueness of the compactification).

In the usual way, there is an abstract notion of Nash manifold, and Nash map, and the corresponding abstract category. Thus we get a subcategory of the analytic and the smooth categories. There is however a caution concerning affine embeddings, which are not always possible, as the example $\mathbb{R} / \mathbb{Z}$ in the preceding section makes clear. We will always discuss affine Nash manifolds, that is, Nash manifolds that can be embedded in some space $\mathbb{R}^{n}$. For these, the above description of Nash functions generalizes as follows:

Artin-Mazur description. Let $M \subset \mathbb{R}^{p}$ be an affine Nash manifold and $f_{1}, \ldots, f_{\ell}$ Nash functions on $M$. Then there exist an algebraic subset $V \subset \mathbb{R}^{k}$, a Nash embedding $\sigma: M \rightarrow \operatorname{Reg}(V)$ and polynomial functions $g_{1}, \ldots, g_{\ell}: \mathbb{R}^{k} \rightarrow \mathbb{R}$ such that $f_{i}=g_{i} \circ \sigma$ for $i=1, \ldots, \ell$.

On the other hand, it is important to remark that while Nash sets are strictly more general than real algebraic sets defined by polynomial equations, the use of inequalities gives nothing but semialgebraic sets defined by inequalities of polynomials; this goes back to the work [L] by Lojasiewicz. Thus the Nash category is in fact inside the semialgebraic category of sets and maps.

Spaces of Nash (or semialgebraic $\mathcal{C}^{r}$ ) maps between Nash manifolds are equipped with their Whitney topologies, defined through approximation of a function with its derivatives. Here one has to be careful when discussing approximation problems for Nash maps, which are not at all easy, and often the key for further progress. But in the end, the standard basic facts hold true: (i) semialgebraic $\mathcal{C}^{r}$ diffeomorphisms form open sets, (ii) semialgebraic $\mathcal{C}^{r}$ maps can be approximated by Nash maps, and even more, (iii) the approximation can be made relative to any Nash set. All these matters were first studied by Efroymson in [Ef3] and settled completely by Shiota in [Sh1]. 
After this presentation, our topic is the sheaf $\mathcal{N}_{M}$ of germs of Nash functions on an affine Nash manifold $M$. The ring $\mathcal{N}(M)=H^{0}\left(M, \mathcal{N}_{M}\right)$ of global sections of this sheaf is the ring of Nash functions on $M$.

\section{Global properties and cohomological failures}

Let $M \subset \mathbb{R}^{n}$ be a Nash manifold, and for the sake of simplicity, suppose $M$ is closed in $\mathbb{R}^{n}$. All that follows mimics what we know of the sheaves $\mathcal{C}$ and $\mathcal{O}$ of smooth and analytic functions.

The Nash functions on $M$ can be defined in the Whitney style, as the smooth functions $f: M \rightarrow \mathbb{R}$ that extend locally to Nash functions on open sets of $\mathbb{R}^{n}$. This only means that a Nash function on $M$ is a global section of the sheaf $\mathcal{N}_{M}=\mathcal{N}_{\mathbb{R}^{n}} / \mathcal{I}_{M}$, where $\mathcal{I}$ is the sheaf of ideals of Nash function germs vanishing on $M$. The question is then whether every Nash function on $M$ is the restriction of a Nash function on $\mathbb{R}^{n}$. This problem is elementary for smooth functions (partitions of unity), and also true for analytic functions (Cartan's Theorems A and B). In the Nash case, however, the extremely subtle proof came out from successive progress by Efroymson [Ef3] and Shiota [Sh1], as we will stress soon. It should be remarked how this extension for Nash submanifolds remains crucial for the whole picture, as far as we know it.

Let us make clear the role of cohomology. Since $\mathcal{N}(M)=H^{0}\left(\mathbb{R}^{n}, \mathcal{N}_{\mathbb{R}^{n}} / \mathcal{I}_{M}\right)$, our question can be reformulated in this way: is the restriction homomorphism $H^{0}\left(\mathbb{R}^{n}, \mathcal{N}_{\mathbb{R}^{n}}\right) \rightarrow H^{0}\left(\mathbb{R}^{n}, \mathcal{N}_{\mathbb{R}^{n}} / \mathcal{I}_{M}\right)$ surjective? Moreover, notice that the kernel of that homomorphism is the ideal $\mathcal{I}(M) \subset \mathcal{N}\left(\mathbb{R}^{n}\right)$ of all Nash functions vanishing on $M$, which is the ideal of global sections of the sheaf $\mathcal{I}_{M}$, and the latter question asks whether the following sequence is exact:

$$
0 \rightarrow H^{0}\left(\mathbb{R}^{n}, \mathcal{I}_{M}\right) \rightarrow H^{0}\left(\mathbb{R}^{n}, \mathcal{N}_{\mathbb{R}^{n}}\right) \rightarrow H^{0}\left(\mathbb{R}^{n}, \mathcal{N}_{\mathbb{R}^{n}} / \mathcal{I}_{M}\right) \rightarrow 0
$$

But this is the sequence of global sections of $0 \rightarrow \mathcal{I}_{M} \rightarrow \mathcal{N}_{\mathbb{R}^{n}} \rightarrow \mathcal{N}_{\mathbb{R}^{n}} / \mathcal{I}_{M} \rightarrow 0$, which is an exact sequence of sheaves with long exact sequence

$$
0 \rightarrow H^{0}\left(\mathbb{R}^{n}, \mathcal{I}_{M}\right) \rightarrow H^{0}\left(\mathbb{R}^{n}, \mathcal{N}_{\mathbb{R}^{n}}\right) \rightarrow H^{0}\left(\mathbb{R}^{n}, \mathcal{N}_{\mathbb{R}^{n}} / \mathcal{I}_{M}\right) \rightarrow H^{1}\left(\mathbb{R}^{n}, \mathcal{I}_{M}\right),
$$

and we would have a positive answer to the question (like in the analytic case by Cartan's Theorem B), if $H^{1}\left(\mathbb{R}^{n}, \mathcal{I}_{M}\right)=0$. Exactly at this point is where Hubbard's counterexample breaks the classical argument, even in the most simple case $H^{1}\left(\mathbb{R}, \mathcal{N}_{\mathbb{R}}\right) \neq 0$ : the cocycle $h=\sqrt{1-x^{2}}$ in $(-\infty, 1) \cap(-1,+\infty)$ represents a nontrivial cohomology class. Indeed, suppose there are Nash functions $f$ and $g$ defined on $(-1,+\infty)$ and $(-\infty,+1)$ respectively, such that $h=f-g$. 


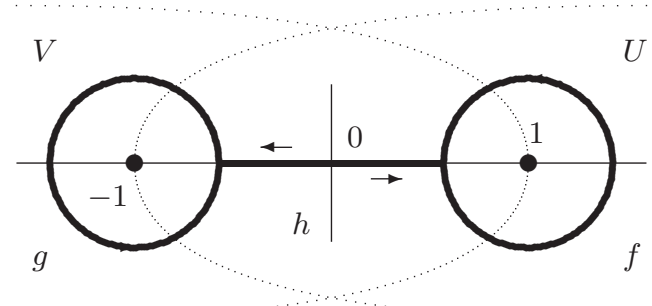

Then, $f$ can be extended to a neighborhood $U$ of the interval $(-1, \infty)$ in the complex plane, $g$ to one $V$ of $(-\infty, 1)$, and $h$ to $U \cap V$, as in the picture above. Now we start with $g$ at 0 following a loop to the right around 1 , then back to 0 , then to the left around -1 and back to 0 . Let us see the result. After the first loop around 1 in $U$, $g=f-\sqrt{1-x^{2}}$ becomes $f+\sqrt{1-x^{2}}$. In $U \cap V$ we have $f=g+\sqrt{1-x^{2}}$, and so $f+\sqrt{1-x^{2}}=g+2 \sqrt{1-x^{2}}$. This latter goes around -1 in $V$ to return to 0 as $g-2 \sqrt{1-x^{2}}$. Hence, $g$ and $g-2 \sqrt{1-x^{2}}$ are two different branches of the same algebraic function. Further turns on the loop give infinitely many more branches $g-4 \sqrt{1-x^{2}}, g-6 \sqrt{1-x^{2}} \ldots$, which is impossible for an algebraic function.

We can also ask whether $\mathcal{N}(M)=H^{0}\left(\mathbb{R}^{n}, \mathcal{N}_{\mathbb{R}^{n}} / \mathcal{I}_{M}\right)$ is really the good choice for the ring of Nash functions on $M$. To illustrate this, consider the set

$$
X: h=z\left(x^{2}+y^{2}\right)-x^{3}=0 .
$$

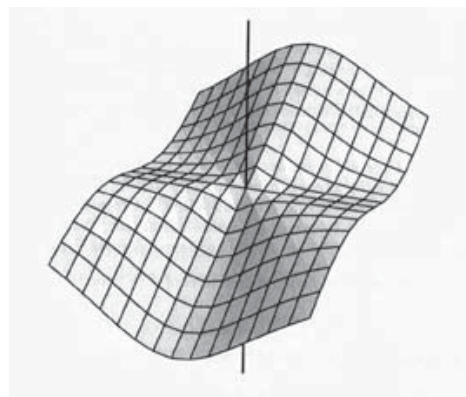

This is a singular surface of $\mathbb{R}^{3}$ consisting of a pure 2-dimensional cloth and the $z$ axis. Efroymson showed that the function

$$
f(x, y, z)= \begin{cases}\frac{(z-1)^{2}}{(z-1)^{2}+x^{2}+y^{2}} & \text { on the cloth } \\ 1 & \text { on the } z \text { axis }\end{cases}
$$

cannot be extended to a Nash function. Indeed, if $f$ had such an extension to some neighborhood of $X$ in $\mathbb{R}^{3}$, it would have an holomorphic extension $f_{\mathbb{C}}$ to some complex neighborhood of the analytic complexification $X_{\mathbb{C}}$ of $X$. Looking at the complex points $(x, x \sqrt{x-1}, 1) \in X_{\mathbb{C}}$ near $(0,0,1)$ we would conclude

$$
1=f(0,0,1)=\lim f_{\mathbb{C}}(x, x \sqrt{x-1}, 1)=0,
$$


a contradiction. In other words, there is an obstruction at the point $(0,0,1)$ along the complex branches of $X$. What happens is that $f$ is not a global section of the sheaf $\mathcal{N}_{\mathbb{R}^{3}} / h \mathcal{N}_{\mathbb{R}^{3}}$.

Note here that we do not work modulo the sheaf of ideals $\mathcal{I}_{X}$ of germs vanishing on $X$. This is something we already learnt from Cartan. In the real case there are anomalous phenomena caused by lack of coherence: this example $X$, called in fact Cartan's umbrella, is not coherent at the origin. Cartan discovered that this difficulty should be avoided by looking at the biggest coherent sheaf of ideals whose zero set is $X$. This coherent sheaf $\mathcal{J}_{X}$ is the right choice to replace $\mathcal{I}_{X}$. For Cartan's umbrella, $\mathcal{J}_{X}=h \mathcal{N}_{\mathbb{R}^{3}}$; for a Nash manifold this coherent sheaf coincides with the sheaf of ideals vanishing on the manifold, property expressed by saying that (real) manifolds are always coherent.

Clearly, all the above explanations involve the search of global equations. As said before, the first author that brought this matter to consideration was Efroymson ([Ef3],[Ef4]), who obtained some results whose hypotheses included assumptions similar to this: let $M$ be a Nash manifold of the form $h=0$ for some Nash function $h$ defined in an open neighborhood of $M$... Actually, the final step added by Shiota ([Sh1]) to complete the solution to the extension problem for $M$ was to show that such a global equation $h$ always exists. This is easy in the smooth category (partitions of unity again), and true in the analytic category (Cartan's again), but not at all in the Nash category. Using sheaves, if $\mathcal{J}_{M}=\left(h_{1}, \ldots, h_{r}\right) \mathcal{N}_{\mathbb{R}^{n}}$ for suitable global sections $h_{i} \in \mathcal{I}(M)$, we could take $h=h_{1}^{2}+\cdots+h_{r}^{2}$. This leads to Cartan's Theorem A, which says when a sheaf is generated by its global sections. In fact, we also need that $\mathcal{N}\left(\mathbb{R}^{n}\right)$ is a noetherian ring, to find finitely many global generators. This property of $\mathcal{N}\left(\mathbb{R}^{n}\right)$, far from evident, was proved around the time Hubbard found his counterexample ([Ef1],[Ri]). Furthermore, one more ingredient is essential in the solutions of the extension problem by Efroymson and Shiota, and that is approximation. In fact, Efroymson argument uses approximation by Nash functions in the continuous Whitney topology, while Shiota's improvement mainly dwells on approximation with derivatives.

Finally, there is a third problem of a clear topological nature: the possibility of separating connected components. To give an example, consider the planar algebraic curve $X: h=y^{2}-x\left(x^{2}-1\right)=0$.

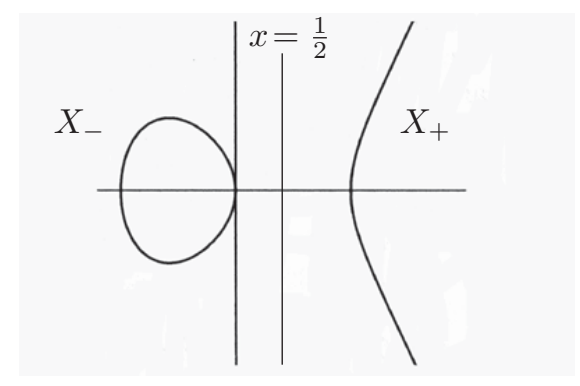


This curve has two connected components $X_{+}$and $X_{-}$, contained in the halfplanes $x>\frac{1}{2}$ and $x<\frac{1}{2}$, respectively. A polynomial that vanishes on any of these components vanishes on the other too (the curve is irreducible); but let us look at the Nash functions $f_{ \pm}=(2 x-1) \mp \sqrt{(2 x-1)^{2}+h^{2}}$. What happens is that $f_{+} \mid X=$ $(2 x-1)-|2 x-1|$ vanishes exactly on $X_{+}$and $f_{-} \mid X$ vanishes exactly on $X_{-}$. This example shows two characteristic features of irreducible algebraic sets in the real case: (i) they need not be connected, and (ii) they need not be irreducible from the Nash viewpoint. About the first fact, a quite general result was soon proved: if an ideal $\mathfrak{p} \subset \mathcal{N}\left(\mathbb{R}^{n}\right)$ is prime, its zero set is connected ([Ri], at the time noetherianness was established). Another basic property is that the connected components can always be separated by means of Nash functions (Mostowski $[\mathrm{Mo}]$ ): notice how we used in the last example the function $2 x-1$, which is $>0$ on $X_{+}$and $<0$ on $X_{-}$. Concerning irreducibility, the immediate comparison is with the analytic category: if a Nash set is irreducible, is it also analytically irreducible?

\section{Formulation of the problems}

Let $M$ be a Nash manifold and $\mathcal{N}_{M}$ its sheaf of germs of Nash functions. The first problem is easy to formulate, and generalizes to germs at the real part of the complexification the last question in the preceding section:

Separation Problem. If $\mathfrak{p} \subset \mathcal{N}(M)$ is a prime ideal, is the ideal $\mathfrak{p O}(M)$ also prime?

For principal ideals, this problem can be stated as follows:

Factorization Problem. If a Nash function $f \in \mathcal{N}(M)$ has a factorization $f=g_{1} g_{2}$ as a product of two analytic functions $g_{1}, g_{2} \in \mathcal{O}(M)$, has it a similar factorization $f=f_{1} f_{2}$ as a product of two Nash functions $f_{1}, f_{2} \in \mathcal{N}(M)$ such that $g_{i}=u_{i} f_{i}$ ?

(It must be stressed that the local versions (for germs) of these problems are easy consequences of M. Artin's approximation theorem.)

While these two problems on reducibility are immediate to state, concerning sheaves we must be very careful. We must bear in mind the extension and global equation problems for Nash submanifolds as presented above, but care about the delicate aspects behind the scenes.

To start with, the first Oka theorem holds for the structural sheaf $\mathcal{N}$, and this reduces coherence to local finite presentation for sheaves of modules, and local finite generation for sheaves of ideals. In other words, a sheaf of ideals $\mathcal{I}$ is coherent, when there is an open covering $\left\{U_{i}\right\}$ of $M$ such that every restriction $\mathcal{I} \mid U_{i}$ is generated by finitely many sections $h_{1}, \ldots, h_{r_{i}} \in H^{0}\left(U_{i}, \mathcal{I}\right)$. However, in contrast to the analytic case, this does not guarantee that $\mathcal{I}$ is generated by its global sections. For instance, let $\mathcal{I}$ be the sheaf of germs of Nash functions vanishing on a discrete set of points 
$x_{k} \in \mathbb{R}$. On a neighborhood of every $x_{k}$ the sheaf is generated by the function $h=x-x_{k}$, (and near the others by $h \equiv 1$ ). Hence, $\mathcal{I}$ is coherent, but it has no non-trivial global section: if $h \in H^{0}(\mathbb{R}, \mathcal{I})$, then $h$ is a Nash function with infinitely many zeros, hence $h \equiv 0$ (this observation is already in [LaTo]). This explains why we must consider a special subclass of coherent sheaves:

Definition. A sheaf of ideals $\mathcal{I} \subset \mathcal{N}$ is called finite when there is a finite covering $\left\{U_{i}\right\}$ of $M$ by open semialgebraic sets, such that every restriction $\mathcal{I} \mid U_{i}$ is generated by finitely many sections $h_{i 1}, \ldots, h_{i r_{i}} \in H^{0}\left(U_{i}, \mathcal{I}\right)$.

Using this notion we can state properly Problems A and B for Nash functions. Let $\mathcal{I} \subset \mathcal{N}_{M}$ be a finite sheaf of ideals.

Problem A: Existence of Global Equations. Is the sheaf $\mathcal{I}$ generated by its global sections $H^{0}(M, \mathcal{I})$ ?

Problem B: Extension. Is the canonical homomorphism

$$
H^{0}\left(M, \mathcal{N}_{M}\right) \rightarrow H^{0}\left(M, \mathcal{N}_{M} / \mathcal{I}\right)
$$

surjective?

It is important to understand the difference stressed by the notion of finiteness, because that is one of the most delicate aspects for the solutions of the problems.

Actually, this finiteness limitation was in fact proposed by Brumfiel more that 20 years ago $([\mathrm{Br}])$. The main idea is: since the usual topology is generated by the open balls, which can be described by strict polynomial inequalities, we restrict ourselves to open sets which can be described by such inequalities, open sets that are called open semialgebraic. The point to care of is that not every union of open semialgebraic sets is again open semialgebraic, and consequently only finite unions are allowed. Thus we speak of the semialgebraic topology; this is the topology used for abstract semialgebraic spaces in [DfKn]. In other words, this semialgebraic topology is the Grothendieck topology whose coverings are generated by finite coverings by open semialgebraic sets. The finite sheaves are those which are locally finitely generated with respect to this semialgebraic topology.

Thus, there are two different sheaves of Nash funtions: one is $\mathcal{N}_{M}$ with respect to the usual topology, and the other is $\mathcal{N}_{M}^{\text {sa }}$ with respect to the semialgebraic topology; in fact, to every sheaf $\mathcal{P}$ for the usual topology we can associate another $\mathcal{P}^{\text {sa }}$ for the semialgebraic topology. Clearly in dealing with the semialgebraic topology we must consider finite sheaves, instead of merely coherent sheaves, but there is a more subtle (and critical) distinction. If $\mathcal{I} \subset \mathcal{N}_{M}$ is a finite sheaf of ideals, we do not know whether the global sections of $\left(\mathcal{N}_{M} / \mathcal{I}\right)^{\text {sa }}$ coincide with those of $\mathcal{N}_{M}^{\mathrm{sa}} / \mathcal{I}^{\mathrm{sa}}$. In other words, a section of $\mathcal{N}_{M} / \mathcal{I}$ for the usual topology is defined over an arbitrary covering, and although we can always choose the members of that covering to be open semialgebraic, it is far from clear that in addition the covering itself can be chosen 
finite. To measure the importance that this finiteness of global sections difficulty has in the framework of all problems involved, we notice here that solving it for a sheaf $\mathcal{I}$ of radical ideals is a first essential step for separation, and that the difficulty can be solved for arbitrary $\mathcal{I}$ only when extension is settled.

On the other hand, the semialgebraic topology is not a true topology (arbitrary unions of open sets are missing), and this can make the situation awkward. Of course, all these discussions are irrelevant if the manifold $M$ is compact. In any case, there is an efficient way of moving to a compact space where the semialgebraic topology becomes a true topology. Or, equivalently, there is a way to compactify $M$ with the semialgebraic topology: this compactification $\widetilde{M}$, called the real spectrum, is the Stone space associated to the family of open semialgebraic sets of $M$.

\section{Solutions in the compact case}

Let us suppose now that the manifold $M$ is compact. As was said before, the difficulties around finiteness vanish, which makes the discussion simpler. Another advantage of the compact case is that analytic functions are easier to treat globally. This comes from the fact that the canonical inclusion $\mathcal{N}(M) \rightarrow \mathcal{O}(M)$ is a regular homomorphism of excellent rings, technical terms from Commutative Algebra; in more down to earth words, both rings are noetherian, their maximal ideals are associated to the points of $M$, and for each of them there is a local system of coordinates $\left(x_{1}, \ldots, x_{m}\right)$ by global Nash functions $x_{i}: M \rightarrow \mathbb{R}$. By these properties, we can apply general Néron's desingularization, a deep theorem of Algebraic Geometry, conjectured by Artin. The consequence is that in the compact case we can do more, and prove the following global version of a local fundamental theorem due (again) to Artin ([Ar]):

Approximation Theorem. Let $\mathrm{y}=\left(\mathrm{y}_{1}, \ldots, \mathrm{y}_{p}\right)$ be new indeterminates, and consider $F_{1}, \ldots, F_{q} \in \mathcal{N}(M)[\mathrm{y}]$. Then every analytic solution $y_{i}=f_{i}(x) \in \mathcal{O}(M), 1 \leq$ $i \leq p$, of the system

$$
F_{1}(x, y)=\cdots=F_{q}(x, y)=0
$$

can be approximated in the Whitney topology by a Nash solution $y_{i}=g_{i}(x) \in \mathcal{N}(M)$, $1 \leq i \leq p$.

In the Nash labyrinth diagram, this theorem is more specifically named global Nash approximation of analytic solutions of polynomial equations. In fact the result also holds for Nash equations, that is, for $F_{1}, \ldots, F_{q} \in \mathcal{N}\left(M \times M^{\prime}\right)$ and $y=f(x) \in$ $\mathcal{O}\left(M, M^{\prime}\right)$. This follows of course by using extension and global equations for $M^{\prime}$ embedded in some $\mathbb{R}^{p}$. Although for compact manifolds this is not needed, we point out that this particular case of the problems will be necessary to complete the solution in the non-compact case. As remarked before, this important case was brought into attention by Efroymson and his arguments completed by Shiota. A variation of this approximation theorem where singularities on the domain are allowed was deduced in [TaTo2]. 
With this theorem as starting step, we cover the following piece of the Nash labyrinth:

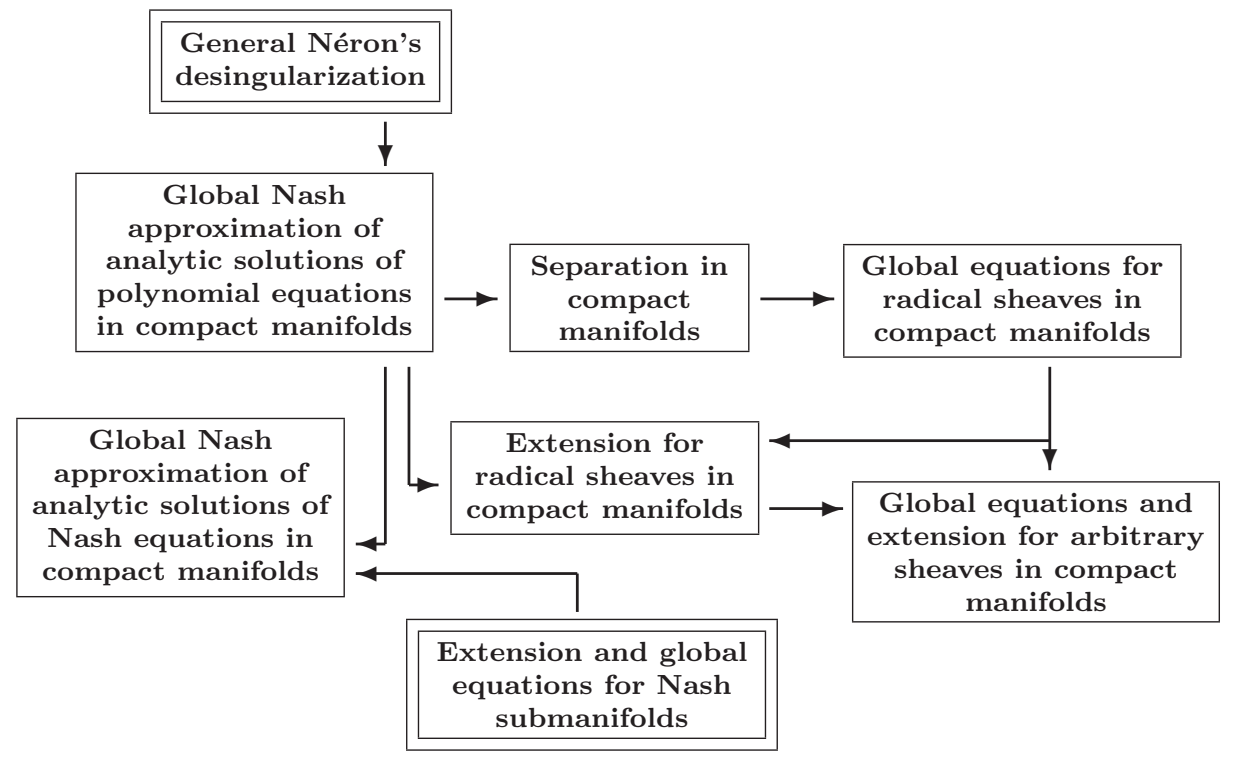

Let us describe, at least briefly, these steps, presented and developed in [CoRzSh1].

Proof of the approximation theorem. We remark first that general Néron's desingularization has been a controversial matter for many years. In the middle 80's, Popescu published a proof followed by several revisions to convince the specialists ([Po]), mostly skeptic we must say. Somehow later, Rotthaus published in [Rt] a very important local partial result. Finally, in the early 90's André ([An]) revisited and completed Popescu's work, and, independently, Spivakovsky ([Sp]) found a different proof. It is good to mention here [Qz1], which is the most readable presentation by far (for rings containing $\mathbb{Q}$ ), and even has some relevant refinements concerning complete intersections. In addition we refer to the survey by Teissier in the Séminaire Bourbaki ([Te]), where also the connections with Nash functions are described. In our case, Néron desingularization says that the regular homomorphism $\mathcal{N}(M) \rightarrow \mathcal{O}(M)$ is a direct limit of smooth finitely generated homomorphisms, an algebraic counterpart of submersions as étale was of local diffeomorphism. This means that the morphism $\mathcal{N}(M)[\mathrm{y}] /\left(F_{1}, \ldots, F_{q}\right) \rightarrow \mathcal{O}(M)$ which sends y to the analytic solution $f=\left(f_{1}, \ldots, f_{p}\right)$ factors through a regular algebra $B=$ $\mathcal{N}(M)[\mathbf{z}] /\left(G_{1}, \ldots, G_{s}\right) \rightarrow \mathcal{O}(M)$, with new indeterminates $\mathbf{z}=\left(\mathbf{z}_{1}, \ldots, \mathbf{z}_{r}\right)$ and functions $G_{\ell}$. This exactly says that there are analytic functions $z_{k}=h_{k}(x) \in \mathcal{O}(M)$ such that $G_{\ell}\left(x, h_{1}(x), \ldots, x, h_{r}(x)\right) \equiv 0$, and $f_{i} \in \mathcal{N}(M)\left[h_{1}, \ldots, h_{r}\right] \subset \mathcal{O}(M)$. Hence, $f_{i}(x)=\varphi_{i}\left(x, h_{1}(x), \ldots, h_{r}(x)\right)$ with $\varphi_{i} \in \mathcal{N}(M)[\mathbf{z}], 1 \leq i \leq p$.

Consequently, we have the following commutative diagram 


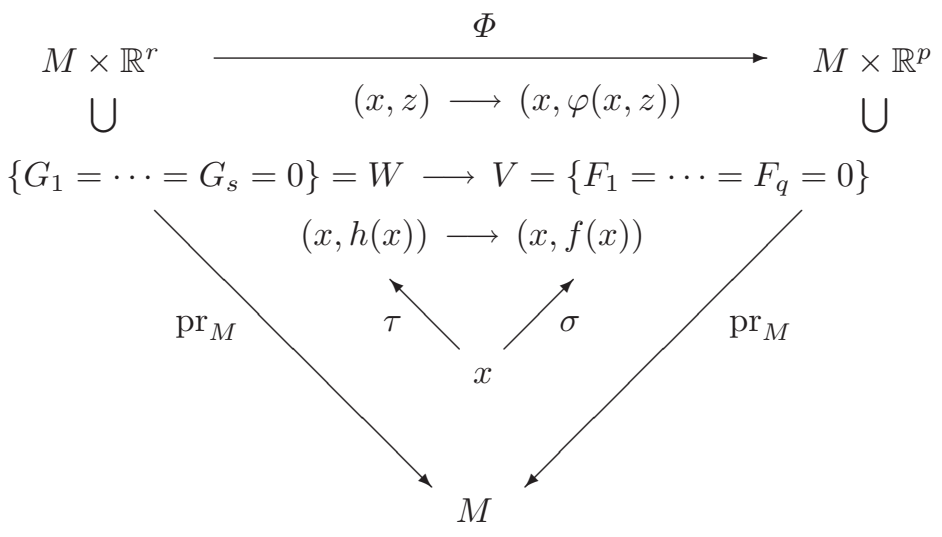

We have thus replaced the analytic solution $y=f(x)$ of the initial Nash system $F=0$ by an analytic solution $z=h(x)$ of the Nash system $G=0$, both represented by sections $\sigma, \tau$ of the canonical projections $V \rightarrow M, W \rightarrow M$ defined on the respective zero sets. The difference is that now, $\mathcal{N}(M)[z] /\left(G_{1}, \ldots, G_{s}\right)$ being a regular algebra, $W$ is a Nash manifold and $W \rightarrow M$ a submersion. Hence, we can approximate $\tau: M \rightarrow W$ (in the Whitney topology) by a Nash map $\theta=\left(\theta_{0}, \theta^{\prime}\right): M \rightarrow W \subset M \times \mathbb{R}^{r}$ (one takes a tubular neighborhood of $W$ in $\mathbb{R}^{m} \times \mathbb{R}^{r}$, and applies the Stone-Weierstrass theorem). Since $\theta_{0}$ is close to the first component of $\tau$, which is the identity, we may assume that $\theta_{0}: M \rightarrow M$ is a diffeomorphism, whose inverse we denote by $\lambda$. Using the Nash maps $\varphi(x, z), \theta(x)$ and $\lambda(x)$ we finally get a Nash solution of the system $F=0$, namely

$$
y=\varphi\left(x, \theta^{\prime}(\lambda(x))\right) .
$$

Indeed, this is a solution because

$$
(x, y)=\left(x, \varphi\left(x, \theta^{\prime}(\lambda(x))\right)\right)=\Phi\left(x, \theta^{\prime}(\lambda(x))\right)=\Phi \theta(\lambda(x)) \in V .
$$

Moreover, as $\theta$ is close to $\tau$ and $\lambda$ is close to the identity, we conclude that $(x, y)$ is close to $\Phi \tau(x)=\sigma(x)=(x, f(x))$.

Proof of separation. Let $\mathfrak{p} \subset \mathcal{N}(M)$ be an ideal and suppose that the extended ideal $\mathfrak{q}=\mathfrak{p} \mathcal{O}(M)$ is not prime. Then there are two analytic functions $f, g \notin \mathfrak{q}$ such that $f g \in \mathfrak{q}$. To formulate this with equations, we choose generators $H_{1}, \ldots, H_{r} \in \mathcal{N}(M)$ of $\mathfrak{p}$, and there will be analytic functions $h_{1}, \ldots, h_{r} \in \mathcal{O}(M)$ such that

$$
f g=h_{1} H_{1}+\cdots+h_{r} H_{r} .
$$

Seeing this as a Nash equation in $2+r$ indeterminates with the analytic solution $f, g, h_{1}, \ldots, h_{r}$, the approximation theorem gives a Nash solution $f^{\prime}, g^{\prime}, h_{1}^{\prime}, \ldots, h_{r}^{\prime}$, so that $f^{\prime} g^{\prime} \in \mathfrak{p}$. But $f^{\prime}$ is close to $f \notin \mathfrak{q}$ in the Whitney topology, for which the ideals are closed, and we conclude $f^{\prime} \notin \mathfrak{q}$, hence $f^{\prime} \notin \mathfrak{p}$. Similarly, $g^{\prime} \notin \mathfrak{p}$, and this means that $\mathfrak{p}$ is not prime. 
Proof of the existence of global equations for radical sheaves. Let $\mathcal{I} \subset \mathcal{N}$ be a finite sheaf of ideals whose stalks $\mathcal{I}_{x}$ are radical ideals. Then the ideal of global sections $I=H^{0}(M, \mathcal{I})$ is also radical, and we must see that $\mathcal{I}=I \mathcal{N}$. To that end, we consider the analytic sheaf $\mathcal{J}=\mathcal{I} \mathcal{O}$, which we surely know is generated by its global sections $J=H^{0}(M, \mathcal{J})$, that is: $\mathcal{J}_{x}=\mathcal{I}_{x} \mathcal{O}_{x}=J \mathcal{O}_{x}$ for every $x \in M$ (Cartan's Theorem A). Fix now a point $a \in M$ and consider

$$
I \subset I(a)=\mathcal{I}_{a} \cap \mathcal{N}(M), J \subset J(a)=\mathcal{J}_{a} \cap \mathcal{O}(M) .
$$

The ideal $J(a)$ may not generate $\mathcal{J}$, but it indeed generates $\mathcal{J}$ in some neighborhood of $a$. We claim that $I(a) \mathcal{O}(M)=J(a)$, which concludes the argument, since then: $I(a)$ generates $\mathcal{I} \mid U^{a}$ on some neighborhood $U^{a}$ of $a$ and by compactness $M=U^{a_{1}} \cup$ $\cdots \cup U^{a_{s}}$, from which it can be deduced $I=I\left(a_{1}\right) \cap \cdots \cap I\left(a_{s}\right)$ and $I \mathcal{O}(M)=J$. Whence, let us see that $I(a) \mathcal{O}(M)=J(a)$ to stress the role of separation in this proof. Firstly we have the primary decomposition $\mathcal{I}_{a}=\mathfrak{p}_{1} \cap \cdots \cap \mathfrak{p}_{r}$ in $\mathcal{N}_{a}$, which lies over another $I(a)=\mathfrak{P}_{1} \cap \cdots \cap \mathfrak{P}_{r}$ in $\mathcal{N}(M)$ where $\mathfrak{P}_{i}=\mathfrak{p}_{i} \cap \mathcal{N}(M)$. As the ideals $\mathfrak{P}_{i}$ are prime, by separation the ideals $\mathfrak{Q}_{i}=\mathfrak{P}_{i} \mathcal{O}(M)$ are prime too, and we have

$$
J(a) \supset I(a) \mathcal{O}(M)=\mathfrak{Q}_{1} \cap \cdots \cap \mathfrak{Q}_{r} .
$$

On the other hand $\mathcal{J}_{a}=\mathfrak{q}_{1} \cap \cdots \cap \mathfrak{q}_{r}$ in $\mathcal{O}_{a}$, where each $\mathfrak{q}_{i}=\mathfrak{p}_{i} \mathcal{O}_{a}$ is prime (this is local separation), and setting $\mathfrak{Q}_{i}^{\prime}=\mathfrak{q}_{i} \cap \mathcal{O}(M)$ we conclude

$$
J(a)=\mathfrak{Q}_{1}^{\prime} \cap \cdots \cap \mathfrak{Q}_{r}^{\prime} \supset \mathfrak{Q}_{1} \cap \cdots \cap \mathfrak{Q}_{r} .
$$

But $\mathfrak{Q}_{i} \subset \mathfrak{Q}_{i}^{\prime}$, and both ideals are prime and have the same height (that of $\mathfrak{p}_{i}$ ), which implies they coincide.

Proof of extension for radical sheaves. Let again $\mathcal{I} \subset \mathcal{N}$ be a sheaf of radical ideals, and let us see that the canonical homomorphism $H^{0}(M, \mathcal{N}) \rightarrow H^{0}(M, \mathcal{N} / \mathcal{I})$ is surjective. Since $\mathcal{I}$ has global equations, $\mathcal{I}=I \mathcal{N}$ with $I=H^{0}(M, \mathcal{I})$, and we know that for the coherent analytic sheaf $\mathcal{J}=I \mathcal{O}$ the homomorphism $H^{0}(M, \mathcal{O}) \rightarrow H^{0}(M, \mathcal{O} / \mathcal{J})$ is certainly surjective (Cartan's Theorem B). Consider then a global Nash section $\sigma \in H^{0}(M, \mathcal{N} / \mathcal{I})$, and, by the preceding remark, choose an analytic function $g \in$ $\mathcal{O}(M)$ such that $g_{x}=\sigma_{x} \bmod I \mathcal{O}_{x}$ for every $x \in M$. Compactness gives a finite open semialgebraic covering $M=U_{1} \cup \cdots \cup U_{r}$ and Nash functions $f_{i} \in \mathcal{N}\left(U_{i}\right)$ such that $f_{i, x}=\sigma_{x} \bmod I \mathcal{N}_{x}$ for every $x \in U_{i}$. In order to simplify the presentation and make clearer the main idea, let us assume here that $I$ is prime. Since Nash functions are algebraic over the polynomials, we have equations

$$
a_{n} f_{i}^{n}+\cdots+a_{0}=0 \bmod I \mathcal{N}\left(U_{i}\right),
$$

where $a_{k} \in \mathcal{N}(M), a_{n} \notin I$. If $I$ were not prime we would have to consider several such equations, one for each associated prime of $I$, and to keep track of the zero divisors $\bmod I$. 
Now, since $g=f_{i} \bmod I \mathcal{O}\left(U_{i}\right)$, what we really have is a Nash equation on each $U_{i}$, which has $g$ as an analytic solution. Multiplying the $r$ equations of the $r$ open sets $U_{i}$, we can assume the Nash equation 1 is defined on the whole manifold $M$, and has the analytic solution $g$. Consequently, we can approximate $g$ by some Nash solution $f$. We claim that 1 has finitely many solutions in $\mathcal{O}(M) / I \mathcal{O}(M)$. Indeed, by the good algebraic properties of the extension $\mathcal{N}(M) \subset \mathcal{O}(M)$, the ideal $I \mathcal{O}(M)$ is radical (in fact, we know from separation that it is prime, but we do not need that much here): an intersection of finitely many prime ideals $\mathfrak{p}_{j} \subset \mathcal{O}(M)$, each lying over $I$, that is, $\mathfrak{p}_{j} \cap \mathcal{N}(M)=I$. Hence $a_{n} \notin \mathfrak{p}_{j}$ and our equation is a non-zero polynomial over the domain $\mathcal{O}(M) / \mathfrak{p}_{j}$ and so has finitely many roots there. Since this works for every $j$, the claim follows readily. From the claim we see that for $f$ close enough to $g$, we in fact have $f=g \bmod I \mathcal{O}(M)$ : if $f^{\prime}$ is a different root, $g-f^{\prime} \notin I \mathcal{O}(M)$ and ideals are closed in the Whitney topology. Hence, we are done in case $I$ is a prime ideal.

Proof of the existence of global equations and extension for arbitrary sheaves. Consider all sheaves $\mathcal{I} \subset \mathcal{N}$ for which some of the two properties fails, and their radicals $\sqrt{\mathcal{I}}$. Consider then the collection of the ideals of global sections of those radicals. This is a collection of ideals of the noetherian ring $\mathcal{N}(M)$, and consequently has a maximal element, say corresponding to a sheaf $\mathcal{I} \subset \mathcal{N}$. Then, both properties hold true for every sheaf $\mathcal{J} \supset \sqrt{\mathcal{I}}$ : otherwise, the ideal of global sections of $\sqrt{\mathcal{J}}$ should be equal to that of $\sqrt{\mathcal{I}}$, by maximality of the latter. But global equations holds for radical sheaves, hence $\sqrt{\mathcal{J}}=\sqrt{\mathcal{I}} \subset \mathcal{J}$, so that $\sqrt{\mathcal{J}}=\mathcal{J}$, and the properties would hold for $\mathcal{J}$. After this, one argues by induction to see that $\mathcal{I}$ would verify the properties too. This induction works over an invariant $\alpha$ defined as follows. Since $\sqrt{\mathcal{I}}$ is radical, it is generated by some global sections $f_{1}, \ldots, f_{r}$. The sheaf $\mathcal{I}$ is among those $\mathcal{I}^{\prime}$ such that $\sqrt{\mathcal{I}^{\prime}} \supset \sqrt{\mathcal{I}}$. For such an $\mathcal{I}^{\prime}$ and every $i=1, \ldots, r$, we must have $f_{i}^{\alpha_{i}+1} \in H^{0}\left(M, \mathcal{I}^{\prime}\right)$ with a minimum $\alpha_{i} \geq 0$, and we define $\alpha\left(\mathcal{I}^{\prime}\right)=\sum_{i} \alpha_{i}$. Clearly, if $\alpha\left(\mathcal{I}^{\prime}\right)=0$, then $\mathcal{I}^{\prime} \supset \sqrt{\mathcal{I}}$, and the properties hold for $\mathcal{I}^{\prime}$, which starts the induction. The step for $\alpha\left(\mathcal{I}^{\prime}\right)>0$, assuming the properties for sheaves with $\alpha<\alpha\left(\mathcal{I}^{\prime}\right)$, is a $d i$ agram chasing using the pull-back along an epimorphism from some free sheaf $\mathcal{N}^{r+s}$ onto $\sqrt{\mathcal{I}^{\prime}}$ (which exists because $\sqrt{\mathcal{I}^{\prime}}$ has global equations) and suitable projections $\mathcal{N}^{k} \rightarrow \mathcal{N}$. At some point one needs extension for the radical sheaf $\sqrt{\mathcal{I}^{\prime}}$. We also stress that this method is general, and does not rely on compactness.

\section{Solutions in the non-compact case}

We now come to the situation where $M$ need not be compact, which, as was already explained, requires a reformulation of the problems, using finite instead of coherent sheaves.

First we notice that the powerful Approximation Theorem that led to the solutions in the compact case is no longer available in the non-compact situation. On the one hand, we cannot apply general Néron's desingularization since the ring of global 
analytic functions on a non-compact manifold is not noetherian. On the other hand, even Nash approximation of analytic diffeomorphisms fails: there are Nash manifolds which are analytically diffeomorphic, but not Nash diffeomorphic [Sh1]. Hence, the attack line had to be different, and the solutions came in two steps: (i) to show that all the problems are equivalent, and (ii) to solve one of them in the non-compact case. The initial ideas towards (i) appeared in [Sh2] and later in [RzSh], before the complete presentation in ([CoRzSh2]); since at that moment all problems were open, the argument had to be more elaborated than in our presentation here. With respect to (ii), we must wait till [CoSh2], where the existence of global equations is proved: first existence over compact sets, which enables one to, second, compactify the problem and, third, deduce existence in the non-compact case from existence in the compact one. It is well remarkable that the existence over compact sets is based on the semialgebraic (version of) Thom's first isotopy lemma, the main result of [CoSh1]. Also, we recall that separation depends on the finiteness of global sections, as we explained when comparing the usual and the Grothendieck topologies.

As before, we depict the corresponding part of the Nash labyrinth:

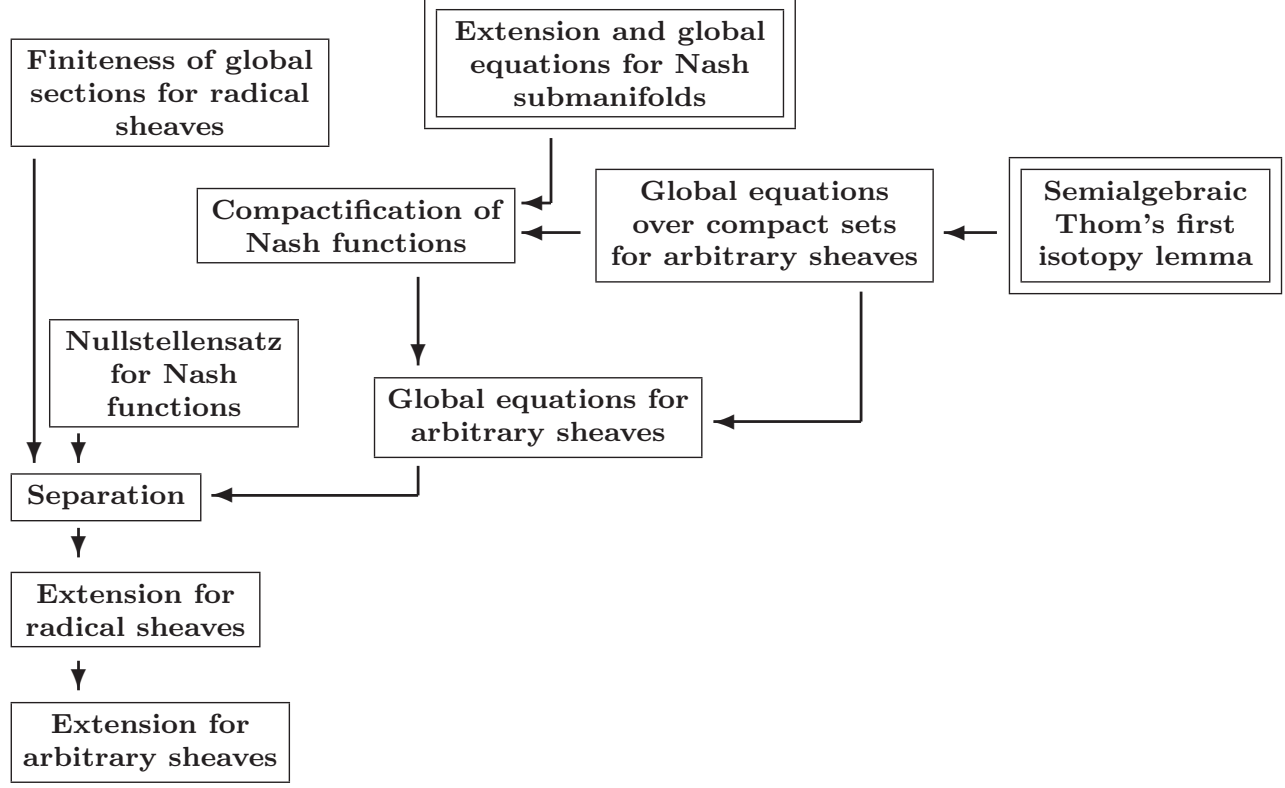

Let us now write precise statements and sketch the proofs of these implications.

Existence of global equations over compact sets. Let $\mathcal{I}$ be a finite sheaf of Nash ideals of $M$, and $Z$ its zero set. For each compact set $K \subset M$ there is an open semialgebraic set $U \supset K \cup(M \backslash Z)$ such that $\mathcal{I} \mid U$ is generated by its global sections $H^{0}(U, \mathcal{I})$. 
Proof. Let $\varphi: M \rightarrow \mathbb{R}$ be a positive proper Nash function. First we prove the weaker assertion where we ask only $U \supset K$. Pick a regular value $r>0$ big enough to have $\varphi \mid K<r$ and define a compact Nash manifold $M_{1} \subset M \times \mathbb{R}$ by $t^{2}=r-\varphi(x)$. The projection $M_{1} \rightarrow M:(x, t) \mapsto x$ induces a Nash diffeomorphism from the open set $U_{1}=\{t>0\}: t=+\sqrt{r-\varphi(x)}$ onto the open set $U \supset K$ given by $r>\varphi(x)$, which transforms $p^{*}(\mathcal{I}) \mid U_{1}$ in $\mathcal{I} \mid U$. But $p^{*}(\mathcal{I})$ is a finite sheaf on the compact Nash manifold $M_{1}$, hence it is generated by some global sections, whose restrictions to $U_{1}$ composed with the diffeomorphism $U \rightarrow U_{1}$ generate $\mathcal{I} \mid U$.

However, we want $U$ bigger, and for this we must improve the construction. Firstly we stratify $Z \subset M$ in a finite union of Nash strata satisfying Whitney's regularity conditions. Then we pick $r>0$ large enough so that $\varphi(K)<r$ and $\varphi:\{\varphi>r\} \rightarrow \mathbb{R}$ is a submersion in restriction to each stratum. Then, by the semialgebraic Thom's first isotopy lemma, we get a semialgebraic homeomorphism (trivialization)

$$
\tau:\{\varphi>r\} \rightarrow\{\varphi=r+1\} \times(r,+\infty): x \mapsto\left(\tau_{1}(x), \varphi(x)\right),
$$

which induces on every stratum $S$ a Nash diffeomorphism

$$
S \cap\{\varphi>r\} \rightarrow(S \cap\{\varphi=r+1\}) \times(r,+\infty),
$$

and $\tau_{1}$ is the identity on $\{\varphi=r+1\}$. Set $V=\{\varphi<r+1\}$. Since $V$ is relatively compact (because $\varphi$ is proper), we can use the weak form of the assertion proved above to deduce that $\mathcal{I} \mid V$ is generated by its global sections. To finish, one defines a Nash diffeomorphism from $V$ onto $U=V \cup(M \backslash Z)$ that transforms $\mathcal{I} \mid V$ in $\mathcal{I} \mid U$. This diffeomorphism depends on the trivialization, of course, but also on a new application of the existence of global equations: the sheaf $\mathcal{I} \mid\{\varphi=r+1\}$ has them because $\{\varphi=r+1\}$ is compact. Indeed, one first constructs a semialgebraic $\mathcal{C}^{1}$ diffeomorphism which is the identity in a neighborhood of $Z \cap V$. Then one approximates it using the Nash approximation theorem relative to a global Nash equation for $Z \cap V$ in $V$.

Here is the place to stress the importance of trivialization results. Notice that in the proof above it is essential that the trivialization is Nash on each stratum, in order to get a Nash diffeomorphism from $V$ onto $U$. The usual proofs of Thom's first isotopy lemma use integration of vector fields and cannot produce trivializations of class Nash. So the use of the semialgebraic version of Thom's isotopy lemma of [CoSh1] is indispensable here. Considerations of this nature are present from the very beginning in the study of semialgebraic sets and maps, back in the pioneering papers by Hardt $([\mathrm{Ha}])$, and remain a matter of high interest. In particular, the so-called Thom's isotopy lemmas are the goal of many recent investigations.

Compactification of Nash functions. Let $f: U \rightarrow \mathbb{R}^{p}$ be a Nash map defined on an open semialgebraic set $U \subset M$, and suppose that $f$ is bounded on a closed semialgebraic set $F \subset U$. Then $M$ is (Nash diffeomorphic to) an open semialgebraic set of an affine Nash manifold $N$, such that the closure of $F$ in $N$ is compact, and $f$ 
extends to a semialgebraic neighborhood $V$ of that closure. In fact, $V \cap M=U$ and $N \backslash M=V \backslash M$.

Proof. We may assume that $M$ is bounded in $\mathbb{R}^{n}$. If $F=M$ we identify $M$ with the graph $\Gamma$ of $f$, which is relatively compact in $\mathbb{R}^{n} \times \mathbb{R}^{p}$, and $f$ is then the linear projection from that graph into $\mathbb{R}^{p}$. In this situation, the candidate for $N$ is the Zariski closure $X$ of $\Gamma$, but that closure may be singular, so that we take for $N$ the desingularization of $X$. This is just a variation of Artin-Mazur's description of the sheaf $\mathcal{N}_{M}$ : every Nash map $M \rightarrow \mathbb{R}^{k}$ is, up to a Nash diffeomorphism, the restriction of a polynomial map $V \rightarrow \mathbb{R}^{k}$ defined on a non-singular real algebraic set $V$ which contains $M$ as an open semialgebraic set.

In general, for arbitrary $F$ one argues as follows. Shrinking $U$ we may suppose that $f$ is in fact bounded on $U$, and there is a bounded Nash function $\sigma$ which is $>1$ on $F$, and $<-1$ on $M \backslash U$ (Mostowski's separation). We can also assume that $M$ is open and relatively compact in a Nash manifold $M_{1}$. Now, we apply the preceding particular case to the map $U \rightarrow M_{1} \times \mathbb{R}^{p+1}: x \mapsto(x, f(x), \sigma(x))$, to get an open inclusion $\varphi: U \rightarrow L$, with $\varphi(U)$ relatively compact in $L$, and an extension $(\eta, g, \bar{\sigma})$ of that map. Let $L^{\prime}$ be the open semialgebraic set of $L$ defined by the inequality $\bar{\sigma}>0$. We obtain the solution by glueing $M$ and $L^{\prime}$ along $\varphi$, but the difficulty is that such a glueing must be made affine.

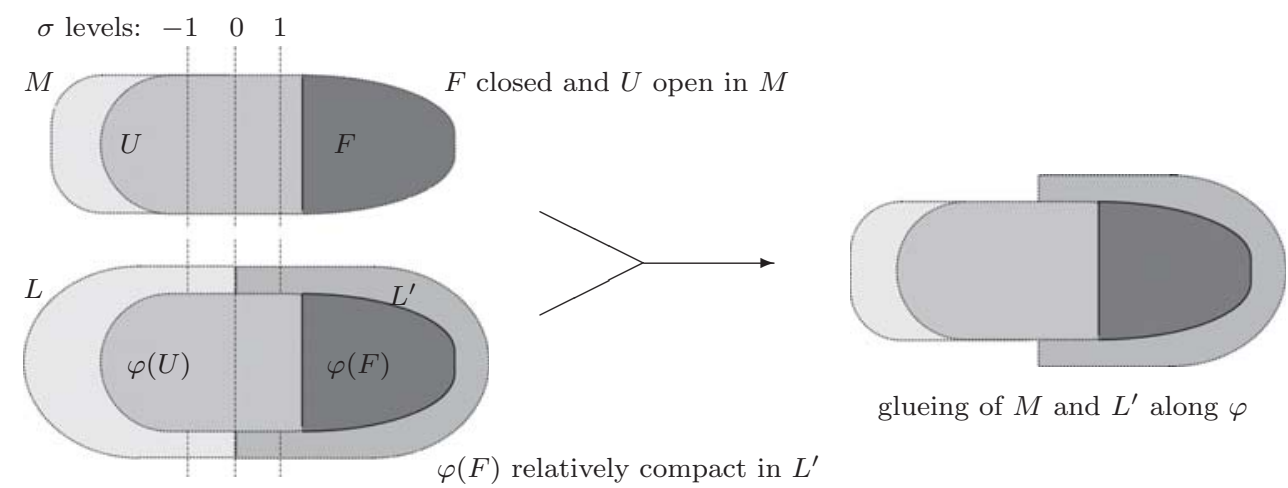

Whence, this glueing must be explicitly constructed in several steps, after a quite technical preparation that uses global equations and extension for Nash submanifolds to control the topological boundary of $\varphi(U)$ in $L$. First, one produces a semialgebraic representation of the glueing of class $\mathcal{C}^{2}$ in some affine space; second, one approximates that $\mathcal{C}^{2}$-representation by a Nash one, say $N$; and third, one approximates the inclusion $M \rightarrow N$, which after the process is no longer Nash. All these approximations are highly technical, and need to keep track of everything as a whole. The third approximation uses Tougeron's improvement of the implicit function theorem. It is of particular importance here to control the jacobian ideal $\mathcal{J}$ of the mapping 
$N \rightarrow M_{1}$ induced by $\eta: L \rightarrow M_{1}$. This $\mathcal{J}$ is a finite sheaf of Nash ideals on $N$, and the existence of global equations over compact sets allows one to assume that $\mathcal{J}$ is generated by its global sections.

Proof of the existence of global equations for arbitrary sheaves. Let $\mathcal{I}$ be a finite Nash sheaf of $M$. By the existence of global equations over compact sets, it suffices to show that $M$ can be identified with a relatively compact open semialgebraic set of another Nash manifold $N$ to which the given sheaf $\mathcal{I}$ extends. It is also clear that it is enough to deal with an $\mathcal{I}$ described by a covering of $M$ consisting of two open semialgebraic sets $U, V: f_{1}, \ldots, f_{k} \in H^{0}(U, \mathcal{I})$ generate $\mathcal{I} \mid U$ and $g_{1}, \ldots, g_{k} \in H^{0}(V, \mathcal{I})$ generate $\mathcal{I} \mid V$. Since each family of functions generates $\mathcal{I} \mid U \cap V$ we have relations

$$
f_{i}=\sum_{j} \alpha_{i, j} g_{j}, g_{i}=\sum_{j} \beta_{i, j} f_{j},
$$

for suitable Nash functions $\alpha_{i, j}, \beta_{i, j}$ defined on $U \cap V$. Hence, what we must do is to extend all these data to $N$. But we have the compactification result to do that, whenever we can bound the involved functions. To bound $f_{i}, g_{i}$ is easy, just replace them by $f_{i} /\left(1+f_{i}^{2}\right)$ and $g_{i} /\left(1+g_{i}^{2}\right)$. But the $\alpha_{i, j}, \beta_{i, j}$ 's require more care. Furthermore, we must compactify without forgetting much of $U, V$. In fact, we consider a separating Nash function $\sigma$ which is $<-1$ on $M \backslash V$ and $>1$ on $M \backslash U$, and add this function to be extended with the others. Now, let $\delta: M \rightarrow \mathbb{R}$ be a positive Nash function that goes to zero at infinity, and such that for some power $\ell$ the products $\delta^{\ell} \alpha_{i, j}, \delta^{\ell} \beta_{i, j}$ are bounded on $-\frac{1}{2} \leq \sigma \leq \frac{1}{2}$ (this is Eojasiewicz's inequality). Notice that the function $\delta$ is a unit in $\mathcal{N}(M)$, hence we can multiply and divide by it at will. Once our data are bounded, we use compactification to extend them to a new Nash manifold $N$ where $M$ is relatively compact. This extension is done in three steps:

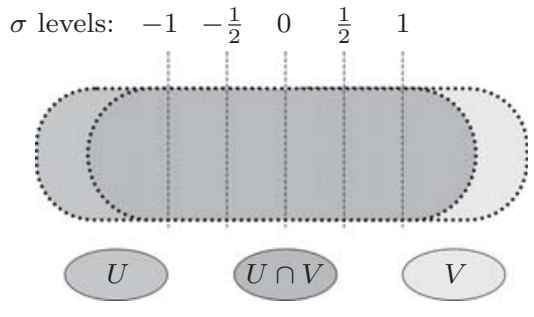

Revista Matemática Complutense 2004, 17; Núm. 1, 83-115
This is the initial $M=U \cup V$, with the $f_{i}$ 's defined on $U$, the $g_{i}$ 's on $V$, the $\alpha_{i j}$ 's $\beta_{i j}$ 's on $U \cap V$, and $\delta, \sigma$ on $U \cup V$. We extend the data to a new Nash manifold where $M$ is relatively compact, or in other words, we extend the data to the limit points of $M$. This is done by three successive applications of the compactification result, proceeding along the levels of the function $\sigma$. 

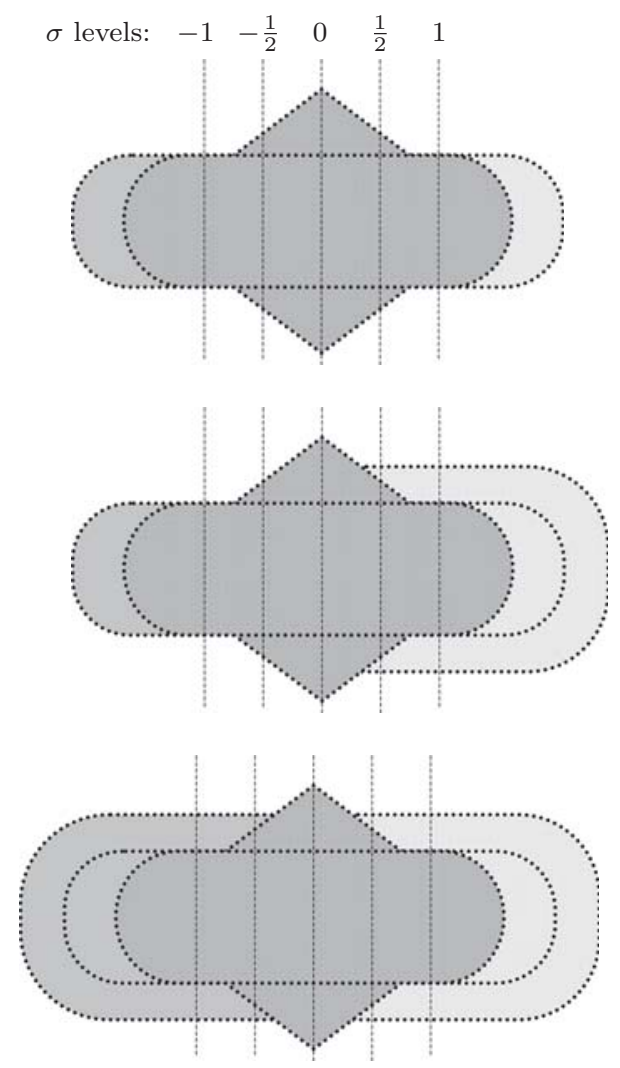

Here we extend to a neighborhood of a part of the limit points of $U \cap V$, including those for $-\frac{1}{2} \leq \sigma \leq \frac{1}{2}$.

Next we extend to a neighborhood of all limit points of $V$ beside $V \backslash U$, including those for $\frac{1}{2} \leq \sigma$.

Finally we extend to a neighborhood of all remaining limit points of $U$, which are beside $U \backslash V$, including those for $\sigma \leq-\frac{1}{2}$.

With all this care, one ends up with a finite sheaf on $N$ that extends $\mathcal{I}$, described in two open semialgebraic sets $U^{\prime}, V^{\prime}$ (whose intersections with $M$ are $U, V$ ) by

$$
\bigcup_{\nu}\left(\left(\bar{f}_{1}, \ldots, \bar{f}_{k}\right): \bar{\delta}^{\nu}\right), \bigcup_{\nu}\left(\left(\bar{g}_{1}, \ldots, \bar{g}_{k}\right): \bar{\delta}^{\nu}\right)
$$

(recall that we multiplied the relations in $U \cap V$ by powers of $\delta$ to bound them, but $\delta$ is a unit on $M$ ). This completes the proof.

Proof of separation. One main tool is a Nullstellensatz for radical finite sheaves. This should be done by complexification, because it is over $\mathbb{C}$ where there is a natural bijection between radical ideals and zero sets. More precisely, one takes a semialgebraic complexification $M^{\mathbb{C}}$ of the manifold $M$, invariant by conjugation, and looks at the germs $X$ along $M$ of complex analytic subsets of $M^{\mathbb{C}}$, specially those invariant by conjugation. Then it can be shown that if $\mathcal{I}$ is a radical finite sheaf, any extension of it to a neighborhood of $M$ in $M^{\mathbb{C}}$ defines such a germ $X=\mathfrak{X}(\mathcal{I})$, which, being semialgebraic, 
has finitely many (invariant) irreducible analytic components $X=X_{1} \cup \cdots \cup X_{r}$. As a matter of fact, we have encountered this earlier, in the proof of extension for radical sheaves in the compact case. There, we could easily remark that a Nash equation had finitely many analytic roots, due to the very nice behaviour of rings of functions in the compact case. Now, we must choose a more geometric view, but then real points would be of little consequence, hence we resource to complex points. Coming back to our analytic components, they are of the form $X_{i}=\mathfrak{X}\left(\mathcal{I}_{i}\right)$ for certain coherent Nash sheaves $\mathcal{I}_{i}$, but it is not evident at all why those $\mathcal{I}_{i}$ 's are to be finite. Suppose that known, and let us see how to deduce separation. A prime ideal $\mathfrak{p} \subset \mathcal{N}(M)$ generates a finite sheaf like $\mathcal{I}$, and the corresponding $\mathcal{I}_{i}$ 's will be generated by their global sections $\mathfrak{p}_{i} \subset \mathcal{N}(M)$ (we already saw that global equations exist). But now, every $X_{i}$ is analytically irreducible, hence $\mathfrak{p}_{i}$ is a prime ideal, and since $\mathfrak{p}=\mathfrak{p}_{1} \cap \cdots \cap \mathfrak{p}_{r}$, there is in fact only one $\mathfrak{p}_{i}$, which must be $\mathfrak{p}$ itself. Thus, $\mathfrak{X}(\mathfrak{p})$ is analytically irreducible, and $\mathfrak{p O}(M)$ is a prime ideal.

Thus, what remains to see is that the $\mathcal{I}_{i}$ 's are indeed finite. It is for this that the real spectrum $\widetilde{M}$ of $M$ is an essential tool. As we already said, $\widetilde{M}$ is a compactification of $M$ whose points $\alpha$ are some ultrafilters of semialgebraic sets of $M$; a point $x \in M$ is represented by the ultrafilter of all semialgebraic sets that contain it. To every semialgebraic set $S \subset M$ there is associated the set $\widetilde{S} \subset \widetilde{M}$ of all $\alpha$ such that $S \in \alpha$. The topology of $\widetilde{M}$ is generated by the sets $\widetilde{U}$, where $U \subset M$ is open semialgebraic. With this topology $\widetilde{M}$ is compact, and $M \subset \widetilde{M}$ inherits the usual topology. The sheaf $\mathcal{N}_{M}$ extends to $\bar{M}$ : the stalk $\mathcal{N}_{\widetilde{M}, \alpha}$ at a point $\alpha$ is the direct limit of the rings $\mathcal{N}(U)$ for all open semialgebraic sets $U \in \alpha$. This ring is a ring of algebraic power series with coefficients in a real closed field $\kappa(\alpha)$ which is determined by (and determines) $\alpha$. A way to describe that real closed field is the following. The Nash functions $f \in \mathcal{N}(M)$ such that $\{f=0\} \in \alpha$ form a prime ideal $\mathfrak{p}_{\alpha}$, and in the quotient field of the domain $\mathcal{N}(M) / \mathfrak{p}_{\alpha}$ there is a total ordering whose positive elements are the classes $f+\mathfrak{p}_{\alpha}$ such that $\{f>0\} \in \alpha$ : after this preparation, $\kappa(\alpha)$ is the real closure of that ordering. Of course, for $\alpha=x \in M$, we have $\kappa(\alpha)=\mathbb{R}$, and $\mathcal{N}_{\widetilde{M}, x}=\mathcal{N}_{M, x}$ is what we already had.

To prove that $\mathcal{I}_{i}$ is finite, by the compactness of $\widetilde{M}$ it suffices to see that for every $\alpha \in \widetilde{M}$ there is an open semialgebraic set $U \subset M$ with $\alpha \in \widetilde{U}$, such that $\mathcal{I}_{i} \mid U$ is generated by its global sections. For this, the following is essential: if $\mathfrak{q} \subset \mathcal{N}_{\widetilde{M}, \alpha}$ is a prime ideal, there is an open semialgebraic set $U \subset M$ and a Nash manifold $S \subset U$ with $\alpha \in \widetilde{S}$, such that for every $x \in S$ the ideal $\mathfrak{q} \mathcal{N}_{M, x}$ is prime. (Notice the small abuse of notation in $\mathfrak{q} \mathcal{N}_{M, x}$.) Such a property was first found in [Hr]; the proof in [CoRzSh2] follows [EkTg] and uses the inverse Rückert-Weierstrass's local parameterization theorem over the field $\kappa(\alpha)$. There is another proof of a more abstract algebraic nature in [Qz3].

We have thus completed the proof of separation, which has needed the following Nullstellensatz: the radical finite sheaves $\mathcal{I}$ correspond bijectively to the invariant analytic germs $X=\mathfrak{X}(\mathcal{I})$ that are locally semialgebraic and have finitely many irreducible components. 
Proof of extension for radical finite sheaves. Let $\mathcal{I}$ be a radical finite sheaf, $X=\mathfrak{X}(\mathcal{I})$ and $\varphi \in H^{0}(M, \mathcal{N} / \mathcal{I})$. We consider the Nash manifold $M^{\prime}=M \times \mathbb{R}$, and write simply $\mathcal{N}=\mathcal{N}_{M}, \mathcal{N}^{\prime}=\mathcal{N}_{M^{\prime}}$. We denote by $(x, t)$ the points of $M^{\prime}$ and define a coherent Nash sheaf $\mathcal{I}(\varphi)$ of $M^{\prime}$ by

$$
\mathcal{I}(\varphi)_{\left(x_{0}, t_{0}\right)}=\left(t-\Phi_{x_{0}}\right)+\mathcal{I}_{x_{0}} \subset \mathcal{N}_{\left(x_{0}, t_{0}\right)}^{\prime},
$$

where $\Phi_{x_{0}} \in \mathcal{N}_{x_{0}}$ induces $\varphi_{x_{0}} \in \mathcal{N}_{x_{0}} / \mathcal{I}_{x_{0}}$. Then $\mathcal{I}(\varphi)$ is radical and finite. Indeed, we can define the germ $X^{\prime}=\mathfrak{X}(\mathcal{I}(\varphi)$ ), which is locally semialgebraic, since the sheaf $\mathcal{I}(\varphi)$ is coherent. On the other hand, as analytic extension is always possible, $X^{\prime}$ is the graph over $X=\mathfrak{X}(\mathcal{I})$ of an analytic function, and exactly as $X$, also $X^{\prime}$ has finitely many irreducible components. By the Nullstellensatz, $X=\mathfrak{X}\left(\mathcal{I}^{\prime}\right)$ for a suitable radical finite sheaf $\mathcal{I}^{\prime}$. But $\mathcal{I}(\varphi)$ is radical as $\mathcal{I}$ is, and again by the Nullstellensatz, we deduce $\mathcal{I}(\varphi)=\mathcal{I}^{\prime}$, and conclude that $\mathcal{I}(\varphi)$ is finite.

Now, since we already know that global equations exist for finite radical sheaves, we can pick generators $f_{1}, \ldots, f_{k}$ of $\mathcal{I}(\varphi)$. Let $\left(x_{0}, t_{0}\right) \in X^{\prime} \cap M^{\prime}$. A standard trick gives units $g_{i} \in \mathcal{N}_{\left(x_{0}, t_{0}\right)}^{\prime}$ such that

$$
g_{i} f_{i,\left(x_{0}, t_{0}\right)}=t-\Phi_{x_{0}} \quad \bmod \mathcal{I}_{x_{0}} \mathcal{N}_{\left(x_{0}, t_{0}\right)}^{\prime} .
$$

We obtain open semialgebraic sets

$$
U_{i}=\left\{(x, t) \in M^{\prime}: t-\Phi_{x} \in\left(f_{i,(x, t)}\right)+\mathcal{I}_{x} \mathcal{N}_{(x, t)}^{\prime}\right\} \quad(1 \leq i \leq k),
$$

that cover $X^{\prime} \cap M^{\prime}$. Explicitly:

$$
\begin{aligned}
U_{i}=p^{-1}(M \backslash X) \cup\left\{(x, t) \in X^{\prime}: \frac{\partial f_{i}}{\partial t}(x, y)\right. & \neq 0\} \cup \\
& \left\{(x, t) \in p^{-1}(M \cap X) \backslash X^{\prime}: f_{i}(x, t) \neq 0\right\} .
\end{aligned}
$$

Using this, one can see that $f_{i}^{-1}(0) \cap U_{i}$ is the graph of a Nash function $F_{i}$ on $V_{i}=p\left(f_{i}^{-1}(0) \cap U_{i}\right)$. On $U_{i}$ the function $f_{i}$ is the product of $t-F_{i}$ by a function without zeros, hence for every $x \in V_{i}$ we have $\varphi_{x}=F_{i, x} \bmod \mathcal{I}_{x}$. The $V_{i}$ 's cover $X$, and the $f_{i}$ 's can be glued by means of a differentiable partition of unity. This glueing is not Nash, but one can combine it with a suitable Nash approximation to get a Nash function $F: M \rightarrow \mathbb{R}$ that represents the section $\varphi$.

To complete the proof one uses the general argument described in the compact case to deduce extension for arbitrary sheaves, once global equations and extension are available for radical sheaves.

\section{Nash functions over arbitrary real closed fields}

All we have explained till now is based on transcendental methods, although we always get results concerning Nash functions, which are defined algebraically using polynomials. Thus the typical question arises: what if instead of the real numbers $\mathbb{R}$ we 
work over an arbitrary real closed field $R$ ? Of course, analytic functions do not make sense in the context, but Nash functions do. Indeed, the latter can be defined either as smooth functions satisfying a non trivial polynomial equation, or using the Artin-Mazur description. Hence, the Existence of Global Equations and Extension problems can be formulated over $R$ as well as over $\mathbb{R}$. We describe here their solutions after [CoRzSh3].

The general strategy for these matters is to apply Tarski-Seidenberg's transfer ([BoCoRo $]$ ), which is conveniently stated in the following form

Uniform bound of complexities. A semialgebraic result can be transferred from a real closed field to another if we can find a uniform bound for the complexities of the semialgebraic objects whose existence is shown, in terms of the complexities of the initial semialgebraic data.

The notion of complexity involved here can be any that verifies some simple general axioms, which in fact give a sensible uniqueness. It is easy to define explicitly a complexity that satisfies our needs. Every semialgebraic set in an affine space is given by a finite system of equalities and/or inequalities (strict and/or not) involving finitely many polynomials. We can take as a measure of complexity for that description the maximum $m$ of the number of equalities, inequalities, polynomials, variables and degrees. The set will then have complexity $\leq m$. For semialgebraic maps, we can consider the complexity of their graphs. Since Nash manifolds are semialgebraic sets, they have a complexity in this sense, and Nash maps too, because they are also semialgebraic. Thus, for a fixed complexity $m$ we have a generic description of all semialgebraic sets of complexity $\leq m$, just writing down all possible systems of $m$ equalities and inequalities involving $m$ polynomials of degree $m$ in $m$ variables, looking at the coefficients as parameters. This can be summarized by saying that all semialgebraic sets of complexity $\leq m$ can be put in a semialgebraic family parameterized over a semialgebraic set $S$. In our case we are really interested in semialgebraic sets that are Nash manifolds, but these turn out to be the same family restricted to a smaller semialgebraic parameter set $T \subset S$, which moreover can be stratified in such a way that each stratum and the family over it are both Nash manifolds. Another fundamental property is that all the families under consideration are in fact defined over the smallest real closed field, the field $\mathbb{R}_{\text {alg }}$ of real algebraic numbers. As explained before, the concrete realization of the complexity measure is not essential, once some axioms are guaranteed; see [Ra] for a different realization depending on the degree of algebraic dependence.

After these preliminaries, the method to obtain uniform bounds is a putting in families strategy. Roughly, given a construction that associates to given initial data $D_{i}$ some final others $E_{j}$, one works as follows:

(i) Every initial datum $D_{i}$ of complexity $\leq m$ is put in a family $\mathcal{D}_{i}$,

(ii) The construction is performed with $\mathcal{D}_{i}$ as initial data, to get final data $\mathcal{E}_{j}$, 
(iii) One checks that every $\mathcal{E}_{j}$ is a family of data $E_{j}$, and that the construction is compatible with the families involved,

(iv) Finally, the complexity of the datum $E_{j}$ is bounded by the complexity of the family $\mathcal{E}_{j}$, which only depends on $m$.

All of this explained, some remarks are in order concerning the meaning of the problems on Nash functions when the ground field is not $\mathbb{R}$ any more. Firstly, the separation problem has no apparent formulation over an arbitrary real closed field $R$, since analytic functions are no longer available: This lack is not essential, and it is in fact possible, although in a very abstract way, to discuss separation over $R$. However, this generalization is better delayed till the next section. Here, we instead consider the change of ground field, when there are two real closed fields $R \subset R^{\prime}$. We have in this case an extension functor called base change that associates to every Nash manifold $M$ over $R$ another $M^{\prime}$ over $R^{\prime}$ (this depends on the obvious fact that every polynomial with coefficients in $R$ is of course a polynomial with coefficients in $R^{\prime}$, and by Tarski-Seidenberg, the functor behaves without surprises). Thus, we have a homomorphism $\mathcal{N}(M) \rightarrow \mathcal{N}\left(M^{\prime}\right)$, which is as good as $\mathcal{N}(M) \rightarrow \mathcal{O}(M)$ when $M$ is a compact Nash manifold over $\mathbb{R}$. For instance, every prime ideal $\mathfrak{p} \subset \mathcal{N}(M)$ generates a prime ideal $\mathfrak{p} \mathcal{N}\left(M^{\prime}\right)$. The deep reason behind this is the following:

Solutions of linear equations with uniform bounds. If $g$ belongs to the ideal generated by $f_{1}, \ldots, f_{k}$, then there are $h_{1}, \ldots, h_{k}$, with complexities bounded solely in terms of the complexities of $g$ and $f_{1}, \ldots, f_{k}$, such that $g=h_{1} f_{1}+\cdots+h_{k} f_{k}$

It is clear how this result gives uniform bounds for all properties that can be formulated using linear equations, and makes Tarski-Seidenberg available to go back and forth via $\mathcal{N}(M) \rightarrow \mathcal{N}\left(M^{\prime}\right)$. For instance, one deduces that this homomorphism is faithfully flat or that it preserves primary decompositions. Concerning the proof, we notice that the study of complexities of solutions of linear equations has deserved a lot of attention for polynomials since Seidenberg's paper [Se]. Thus, the natural idea for Nash functions is to use the Artin-Mazur description to transform them into polynomials. However, this transformation must keep track of complexities, which leads to another uniform bounds result:

Uniform Artin-Mazur description. Given positive integers $p, c, \ell$, there exist positive integers $k, d$ satisfying the following property. Let $M \subset R^{p}$ be an affine Nash manifold of complexity $\leq c$ and $f_{1}, \ldots, f_{\ell}$ Nash functions on $M$ of complexities $\leq c$. Then there exist an algebraic subset $V \subset R^{k}$ whose ideal is generated by $\leq d$ polynomials of degrees $\leq d$, a Nash embedding $\sigma: M \rightarrow \operatorname{Reg}(V)$ of complexity $\leq d$ and polynomial functions $g_{1}, \ldots, g_{\ell}: R^{k} \rightarrow R$ of degrees $\leq d$ such that $f_{i}=g_{i} \circ \sigma$ for $i=1, \ldots, \ell$.

The proof of this is once again an application of the putting in families strategy described above. 
Concerning the other problems, the main point is to understand the sheaf of germs of Nash functions over arbitrary real closed fields. These fields have a unique ordering, and consequently a topology, but it is a little friendly one. Look for instance at the field $R=\mathbb{R}_{\mathrm{alg}}$. The function $\equiv 1$ on $(-\infty, \pi)$ and $\equiv 0$ on $(\pi, \infty)$ is locally (in the order topology) Nash, but it is not Nash globally on $\mathbb{R}_{\text {alg }}$ (because non-zero Nash functions in one variable must have finitely many zeros). This shows that Nash functions do not define a sheaf for the order topology. However, they form one with respect to the semialgebraic topology (defined as for $R=\mathbb{R}$ ). Thus, in $\mathbb{R}_{\mathrm{alg}}$ the semialgebraic topology is not a second option, but the unique choice. As for $R=\mathbb{R}$, the semialgebraic topology is not a true topology, but this is mended by the theory of the real spectrum.

Once all terms involved are well defined, we can apply the put in a family strategy to prove uniform bounds for global equations and extension over $\mathbb{R}$, and then deduce them over arbitrary real closed fields. Thus we have completed this piece of the Nash labyrinth:

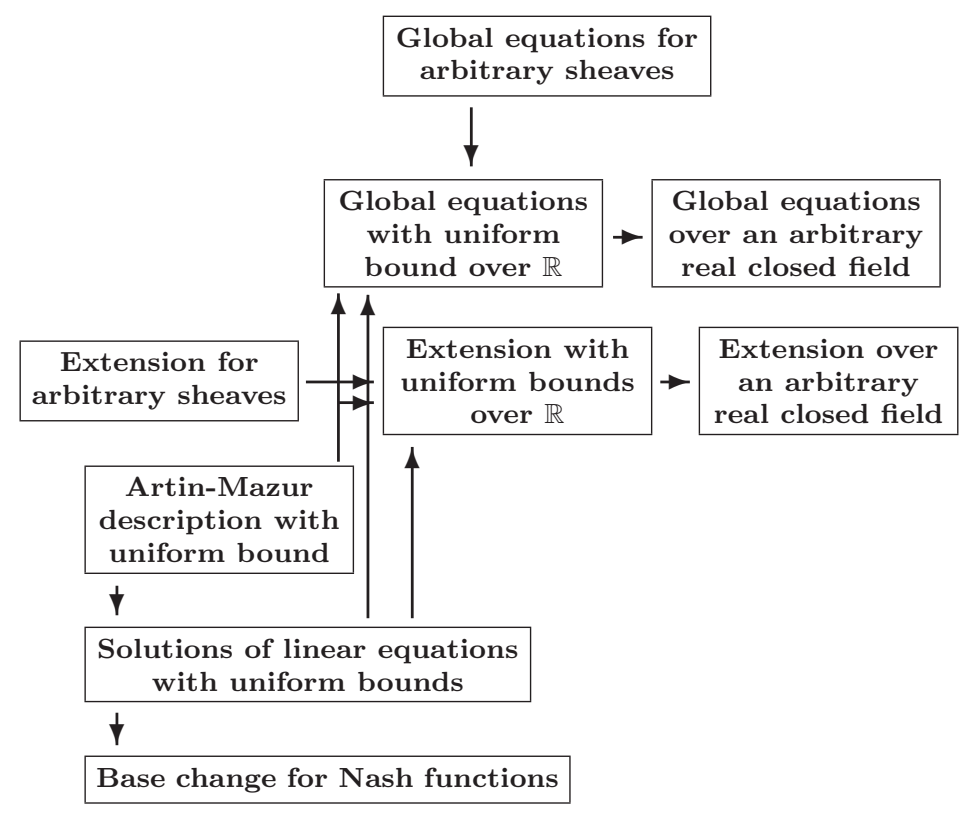

\section{Abstract Nash functions}

Manifolds over arbitrary real closed fields are not the most general setting to define and study Nash functions. Actually, the real spectrum is defined for arbitrary commu- 
tative rings $A$, and for $A=\mathcal{N}(M)$ we get the space $\widetilde{M}$. This is quite natural, recalling how we defined the real closed field $\kappa(\alpha)$. Indeed, for $A$ arbitrary, $\operatorname{Spec}_{r}(A)$ consists of all points $\alpha$ determined by a prime ideal $\mathfrak{p} \subset A$ and a total ordering in the quotient field of $A / \mathfrak{p}$; in other words, we identify $\alpha$ with the homomorphism $A \rightarrow A / \mathfrak{p} \rightarrow \kappa(\alpha)$, where $\kappa(\alpha)$ is the real closure of that ordered field; we write $f(\alpha)$ instead of $\alpha(f)$ for $f \in A$, in order to see the elements of $A$ as functions on $\operatorname{Spec}_{r}(A)$. The set $\operatorname{Spec}_{r}(A)$ is endowed with the topology generated by the basic open sets

$$
\left\{f_{1}>0, \ldots, f_{k}>0\right\}=\left\{\alpha \in \operatorname{Spec}_{r}(A): f_{1}(\alpha)>0, \ldots, f_{k}(\alpha)>0\right\},
$$

with $f_{1}, \ldots, f_{k} \in A$ (and the sign $>$ evaluated in $\kappa(\alpha)$, of course). This gives a compact space. Actually, compactness holds even for the constructible topology, generated by the constructible sets, which are the boolean combinations of the above basic open sets. This construction defines a functor from rings and homomorphisms $f: A \rightarrow B$ to topological spaces and continuous mappings $f^{*}: \operatorname{Spec}_{r}(B) \rightarrow \operatorname{Spec}_{r}(A)$, similar to the Zariski spectrum of modern Algebraic Geometry.

The real spectrum is equipped with the sheaf $\mathcal{N}_{A}$ of germs of Nash functions, copying the definition given for manifolds over $\mathbb{R}$ in $[\mathrm{ArMz}]$. Although it seems too technical, let us state it here. For every open set $U$ of $\operatorname{Spec}_{r}(A)$ consider the direct system of all pairs $(A \rightarrow B, \sigma)$ where $B$ is an étale $A$-algebra and $\sigma: U \rightarrow \operatorname{Spec}_{r}(B)$ is a section of the local homeomorphism $\operatorname{Spec}_{r}(B) \rightarrow \operatorname{Spec}_{r}(A)$, and denote by $(B, \sigma) \rightarrow$ $(C, \tau)$ a homomorphism $f: B \rightarrow C$ of $A$-algebras such that $f^{*} \circ \sigma=\tau$. Then $\mathcal{A}(U)$ is the direct limit of that system; this gives a presheaf $\mathcal{A}$. In case $A=R\left[\mathrm{x}_{1}, \ldots, \mathrm{x}_{n}\right]$ and $U=\widetilde{W}$ for an open semialgebraic set $W \subset R^{n}$, it is $\mathcal{A}(U)=\mathcal{N}(W)$ : this is Artin-Mazur's description of $\mathcal{N}(W)$, and means that $\mathcal{A}$ is a sheaf in this case. The question whether $\mathcal{A}$ is a sheaf for arbitrary $A$ has remained open for many years: this is the Artin-Mazur description for A. Since it was not known to be true, $\mathcal{N}_{A}$ was by definition the sheaf associated to the presheaf $\mathcal{A}$ (of course, everybody thought that $\left.\mathcal{N}_{A}=\mathcal{A}\right)$. This Artin-Mazur description means that:

(i) For every open constructible subset $U$ of $\operatorname{Spec}_{r}(A)$, the ring of Nash functions $\mathcal{N}_{A}(U)$ is an ind-étale $A$-algebra, and

(ii) For every point $\alpha \in \operatorname{Spec}_{r}(A)$, the ring of Nash function germs $\mathcal{N}_{A, \alpha}$ is a local ind-étale A-algebra.

Another important property is idempotency, which was studied at the very moment the real spectrum was invented. Consider the ring $B=\mathcal{N}_{A}\left(\operatorname{Spec}_{r}(A)\right)$ of global sections of $\mathcal{N}_{A}$. Then there is a canonical homomorphism $A \rightarrow B$ which induces a map $\operatorname{Spec}_{r}(B) \rightarrow \operatorname{Spec}_{r}(A)$, and idempotency holds when this map is an isomorphism of ringed spaces, that is, the map is a homeomorphism and transforms $\mathcal{N}_{B}$ in $\mathcal{N}_{A}$. This expresses the stabilization of the construction $A \mapsto \mathcal{N}_{A}\left(\operatorname{Spec}_{r}(A)\right)$ after the first step. This is just natural if we see this construction as a closure or a completion with respect to the implicit function theorem. 
For the proof one defines an inverse $s: \operatorname{Spec}_{r}(A) \rightarrow \operatorname{Spec}_{r}(B)$ as follows: for every $\alpha \in \operatorname{Spec}_{r}(A)$ we have the canonical homomorphism $B \rightarrow \mathcal{N}_{A, \alpha}$, which induces a prime cone $\beta \in \operatorname{Spec}_{r}(B)$. Now, by the general properties of ind-étale limits, $\mathcal{N}_{B, \beta}$ is isomorphic to $\mathcal{N}_{A, \alpha}$, and it remains to see that $s$ is surjective. For many years this was thought to be proved ([Ry],[AlRy]), but the specialists overlooked a gap. It is instructive for our topic the way that gap was discovered. After [CoRzSh1], Quarez found ([Qz2]) an argument that deduced extension from idempotency, by making the ideas in the compact case available in the non-compact one. This lead to a protocolary revision of the quoted articles on idempotency, and to the unexpected finding that the proofs were incomplete. Quarez's work included also the key that the Artin-Mazur description implied idempotency. In fact, Artin-Mazur description was the missing tool to complete the argument above and conclude that our $s$ above is indeed surjective.

Once we have Nash functions on real spectra, we have Nash sheaves too. But, real spectra being compact, finiteness difficulties are excluded: finite sheaf means simply locally finitely generated. Consequently, extension and global equations are formulated readily.

Next we come to the more delicate matter of separation. Again in the paper [Qz3] by Quarez, we find a proposal. It is a Nullstellensatz: $\mathcal{I} \mapsto \mathfrak{X}(\mathcal{I})$ for finite sheaves of ideals in the abstract case. The idea is simple, despite complications coming from the (very interesting but independent) affair of when Nash functions form a noetherian ring. In fact, we can go straight as follows. Let $U \subset \operatorname{Spec}_{r}(A)$ be an open constructible set. For every point $\alpha \in U$ we have the canonical homomorphism $\mathcal{N}_{A}(U) \mapsto \mathcal{N}_{A, \alpha}: f \mapsto f_{\alpha}$ and, looking at the germ at $\alpha$ of the complexification of $U$, the prime ideals $\mathfrak{p} \subset \mathcal{N}_{A, \alpha}$ may be seen as ideals of complex invariant germs. We have then a space $\mathcal{G}$ consisting of all pairs $(\alpha, \mathfrak{p})$. (The idea of abstract complexification is not new: see $[\mathrm{Cu}],[\mathrm{Pu}],[\mathrm{Sc}]$.$) In this space we define$

$$
I \mapsto \mathfrak{X}(I), \quad X \mapsto \mathcal{J}(X),
$$

for ideals $I \subset \mathcal{N}_{A}(U)$ of Nash functions and subsets $X$ of $\mathcal{G}$ as follows:

$$
\begin{gathered}
\mathfrak{X}(I)=\left\{(\alpha, \mathfrak{p}) \in \mathcal{G}: \mathfrak{p} \supset I \mathcal{N}_{A, \alpha}\right\}, \\
\mathcal{J}(X)=\left\{f \in \mathcal{N}_{A}\left(\operatorname{Spec}_{r}(A)\right): f_{\alpha} \in \mathfrak{p} \text { for all }(\alpha, \mathfrak{p}) \in X\right\} .
\end{gathered}
$$

The usual properties of zero sets and ideals of zeros can be easily checked, and we have a topology in $\mathcal{G}$ whose closed sets are those zero sets. Then the abstract Nullstellensatz says that zero sets correspond bijectively to the radical ideals $I$ :

$$
\mathcal{J} \mathfrak{X}(I)=\sqrt{I} .
$$

Proof. This is a consequence of idempotency. Indeed, the thing to check is that $\mathcal{J} \mathfrak{X}(I) \subset \sqrt{I}$. But if $f \in \mathcal{J} \mathfrak{X}(I)$, then $f_{\alpha} \in \mathfrak{p}$ for every $\alpha$ and every prime ideal $\mathfrak{p}$ of $\mathcal{N}_{A, \alpha}$ such that $I \mathcal{N}_{A, \alpha} \subset \mathfrak{p}$, so that $f_{\alpha} \in \sqrt{I \mathcal{N}_{A, \alpha}}$ for every $\alpha$. Now, let $\mathfrak{m}$ be a 
maximal ideal of $\mathcal{N}_{A}(U)$, and $\alpha$ any prime cone supported on $\mathfrak{m}$ (which in fact exists by idempotency). Since the morphism $\mathcal{N}_{A}(U)_{\mathfrak{m}} \rightarrow A_{\alpha}$ is local ind-étale, we deduce that $f \in \sqrt{I \mathcal{N}_{A}(U)_{\mathfrak{m}}}$. This shows that $f \in \sqrt{I}$.

Now, in $\mathcal{G}$ we have the standard notion of irreducibility for the closed sets $\mathfrak{X}(I)$ (although we do not know whether irreducible decompositions exist, since nothing is yet said about noetherianness). Anyway, separation is formulated immediately: if $\mathfrak{p} \subset \mathcal{N}_{A}(U)$ is prime, is the set $\mathfrak{X}(\mathfrak{p})$ irreducible? ? The answer is yes. For let $\mathfrak{X}(\mathfrak{p}) \subset \mathfrak{X}(I) \cup \mathfrak{X}(J)$. Then from the Nullstellensatz we get $\mathfrak{p} \supset \sqrt{I \cap J}$, and $\mathfrak{p}$ being prime, either $\mathfrak{p} \supset I$ or $\mathfrak{p} \supset J$. Hence either $\mathfrak{X}(\mathfrak{p}) \subset \mathfrak{X}(I)$ or $\mathfrak{X}(\mathfrak{p}) \subset \mathfrak{X}(J)$.

In particular, we give a meaning to separation over any real closed field, as was advanced before.

We have thus explained a good part of the remaining implications of the Nash labyrinth:

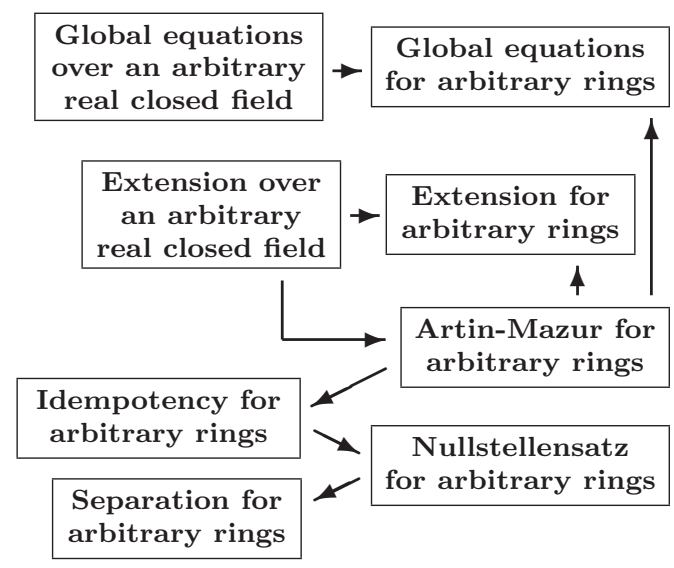

Let us sketch the proofs that are left.

Proof of the Artin-Mazur description for arbitrary rings. Let $\varphi$ be a global section of $\mathcal{N}_{A}$, represented by a finite open covering $\operatorname{Spec}_{r}(A)=U_{1} \cup \cdots \cup U_{r}$ (and finite by the compactness of the real spectrum), with étale algebras $A \rightarrow B_{i}$, continuous sections $\sigma_{i}: U_{i} \rightarrow \operatorname{Spec}_{r}\left(B_{i}\right)$ and elements $f_{i} \in B_{i}$ that verify certain compatibility equations. What we seek is a similar description with a single open set. To that end, we built a commutative ring of the type $A_{0}=\mathbb{Z}\left[\mathrm{x}_{1}, \ldots, \mathrm{x}_{n}\right] /\left(F_{1}, \ldots, F_{s}\right) \subset A$ that contains all involved data. Since $\mathbb{Z} \subset \mathbb{R}_{\mathrm{alg}}$ we can consider the sheaf $\mathcal{N}$ of germs of Nash functions of the affine space $\mathbb{R}_{\text {alg }}^{n}$, and the finite sheaf of ideals $\mathcal{I}$ generated by $F_{1}, \ldots, F_{s} \in \mathbb{Z}\left[\mathrm{x}_{1}, \ldots, \mathrm{x}_{n}\right] \subset \mathcal{N}\left(\mathbb{R}_{\text {alg }}^{n}\right)$. Then our data define a global section of $\mathcal{N} / \mathcal{I}$, and by extension over the real algebraic numbers, that section is represented by a 
global Nash function on $\mathbb{R}_{\text {alg }}^{n}$. But now, by Artin-Mazur's theorem over $\mathbb{R}_{\text {alg, that }}$ global Nash function is in turn represented by an element $f$ of an étale algebra over $\mathbb{R}_{\text {alg }}\left[\mathrm{x}_{1}, \ldots, \mathrm{x}_{n}\right]$. That étale algebra is also étale over $\mathbb{Z}\left[\mathrm{x}_{1}, \ldots, \mathrm{x}_{n}\right]$, and by the change of base $A_{0} \otimes_{\mathbb{Z}}-$ we obtain an étale $A$-algebra. In the end, the element $f$, seen in the latter étale $A$-algebra, represents the section $\varphi$ we started with.

Once the Artin-Mazur description is available for arbitrary rings, Nash functions are described easily, and we can use the same kind of descent argument from $A$ to a finitely generated $\mathbb{Z}$-algebra to prove extension and global equations for sheaves of Nash sections over arbitrary rings.

After this long process of reformulation and generalization, needed to understand the nature of the various problems under view, and the nature of their possible solutions, we have been able to solve them all.

Now, a few words are in order to understand when the rings of Nash functions are noetherian. Firstly, although we have called separation for $\mathcal{N}_{A}(U)$ the irreducibility of the sets $\mathfrak{X}(\mathfrak{p})$, the geometric meaning of this is only clear when the $\operatorname{ring} \mathcal{N}_{A}(U)$ is noetherian. In that case, the space $\mathcal{G}$ is noetherian too, and we have a convincing notion of analytic component as an irreducible closed subset of $\mathcal{G}$, because we have a decomposition into (finitely many) irreducible components. Furthermore, there is a sensible local definition for "analytic sets": let $X \subset \mathcal{G}$ be defined by a finite open constructible covering $U=\bigcup_{i} U_{i}$ and radical ideals $I_{i} \subset \mathcal{N}_{A}\left(U_{i}\right)$ in the form $\left.X\right|_{U_{i}}=\mathfrak{X}\left(I_{i}\right)$ (with the obvious notation). Then the abstract Nullstellensatz implies

$$
I_{i} \mathcal{N}_{A}\left(U_{i} \cap U_{j}\right)=\mathcal{J}\left(\left.X\right|_{U_{i} \cap U_{j}}\right)=I_{j} \mathcal{N}_{A}\left(U_{i} \cap U_{j}\right) .
$$

Thus we have a sheaf of radical ideals $\mathcal{I} \subset \mathcal{N}_{A} \mid U$ defined over each $U_{i}$ by $\left.\mathcal{I}\right|_{U_{i}}=$ $\left.I_{i} \mathcal{N}_{A}\right|_{U_{i}}$. However, we cannot conclude from this that $X=\mathfrak{X}(I)$ for the ideal $I=$ $H^{0}(U, \mathcal{I})$ : global equations exist for finite sheaves, and $\mathcal{I}$ need not be finite. Now, suppose that the rings $\mathcal{N}_{A}\left(U_{i}\right)$ are noetherian. Then each $I_{i}$ is finitely generated, so that $\mathcal{I}$ is in fact finite, and $X$ is a closed set of $\mathcal{G}$.

After these remarks, let us suppose that the $\operatorname{rings} \mathcal{N}_{A}(U)$ are noetherian. First, we can replace $A$ by $A\left[S^{-1}\right]$, where the multiplicative set $S$ consists of all $f \in A$ without zeros in the real spectrum. This makes the ind-étale homomorphism $A \rightarrow \mathcal{N}_{A}(U)$ faithfully flat, and $A$ is noetherian by descent. However, the converse is not always true (counterexamples are easy: take for $A$ the field of a real algebraic curve, which has infinitely many total orderings). Thus, we look for another condition, and find one that had already been discussed in other contexts (see [AnBrRz]), namely, that every constructible set of $\operatorname{Spec}_{r}(A)$ has finitely many connected components. The proof that this is necessary (see [CoRzSh3]) uses the standard tricks for semialgebraic separation, available in the abstract setting after the work of Mahé ([Mh]). Finally, we can close the circle by showing that if $\mathcal{G}$ is noetherian, the $\operatorname{ring} \mathcal{N}_{A}(U)$ is noetherian too. 
Finally, let us state the best positive result known so far (essentially proved in [Qz3]; also proved, assuming idempotency, for finitely generated algebras over real closed fields in $[\mathrm{Cu}])$ :

Proposition. Suppose that $A$ is excellent, and every constructible subset of $\operatorname{Spec}_{r}(A)$ has finitely many connected components. Then the rings $\mathcal{N}_{A}(U)$ of Nash functions on open constructible sets $U \subset \operatorname{Spec}_{r}(A)$ are excellent.

\section{References}

[AlRy] M. E. Alonso and M.-F. Roy, Real strict localizations, Math. Z. 194 (1987), 429-441.

[An] M. André, Cinq exposés sur la désingularisation, École Polythecnique Fédérale de Lausanne, 1992 (manuscript).

[Ar] M. Artin, On the solutions of analytic equations, Invent. Math. 5 (1968), 277-291.

[ArMz] M. Artin and B. Mazur, On periodic points, Ann. of Math. (2) 81 (1965), 82-99.

[AnBrRz] C. Andradas, L. Bröcker, and J. M. Ruiz, Constructible sets in real geometry, Ergebnisse der Mathematik und ihrer Grenzgebiete (3), vol. 33, Springer-Verlag, Berlin, 1996, ISBN 3-54060451-0.

[BeTo] L. Beretta and A. Tognoli, Nash sets and global equations, Boll. Un. Mat. Ital. A (7) 4 (1990), $31-44$.

[BoCoRo] J. Bochnak, M. Coste, and M.-F. Roy, Real algebraic geometry, Ergebnisse der Mathematik und ihrer Grenzgebiete (3), vol. 36, Springer-Verlag, Berlin, 1998, ISBN 3-540-64663-9.

[Br] G. W. Brumfiel, Partially ordered rings and semi-algebraic geometry, London Mathematical Society Lecture Note Series, vol. 37, Cambridge University Press, Cambridge, 1979, ISBN 0$521-22845-\mathrm{X}$

[CoDp] M. Coste and M. M. Diop, On some problems about Nash functions, Univ. Rennes I, 1991 (preprint).

[CoRzSh1] M. Coste, J. M. Ruiz, and M. Shiota, Approximation in compact Nash manifolds, Amer. J. Math. 117 (1995), 905-927.

[CoRzSh2] _ Separation, factorization and finite sheaves on Nash manifolds, Compositio Math. 103 (1996), 31-62.

[CoRzSh3] _ Uniform bounds on complexity and transfer of global properties of Nash functions, J. Reine Angew. Math. 536 (2001), 209-235.

[CoSh1] M. Coste and M. Shiota, Nash triviality in families of Nash manifolds, Invent. Math. 108 (1992), 349-368

[CoSh2] _ Nash functions on noncompact Nash manifolds, Ann. Sci. École Norm. Sup. (4) 33 (2000), 139-149.

[CoSh3] _ Thom's first isotopy lemma: a semialgebraic version, with uniform bound, Real Analytic and Algebraic Geometry (Trento, 1992), de Gruyter, Berlin, 1995, pp. 83-101.

[Cu] F. Cucker, Sur les anneaux de sections globales du faisceau structural sur le spectre réel, Comm. Algebra 16 (1988), 307-323.

[DfKn] H. Delfs and M. Knebusch, Semialgebraic topology over a real closed field. II. Basic theory of semialgebraic spaces, Math. Z. 178 (1981), 175-213. 
[vdD] L. van den Dries, Tame topology and o-minimal structures, London Mathematical Society Lecture Note Series, vol. 248, Cambridge University Press, Cambridge, 1998, ISBN 0-521-598389

[Ef1] G. A. Efroymson, A Nullstellensatz for Nash rings, Pacific J. Math. 54 (1974), 101-112.

[Ef2] _ Substitution in Nash functions, Pacific J. Math. 63 (1976), 137-145.

[Ef3] _ The extension theorem for Nash functions, Real Algebraic Geometry and Quadratic Forms (Rennes, 1981), Lecture Notes in Math., vol. 959, Springer, Berlin, 1982, pp. 343-357.

[Ef4] _ Nash rings on planar domains, Trans. Amer. Math. Soc. 249 (1979), 435-445.

[EkTg] A. El Khadiri and J.-Cl. Tougeron, Familles noethériennes de modules sur $k \llbracket x \rrbracket$ et applications, Bull. Sci. Math. 120 (1996), 253-292.

[Ha] R. M. Hardt, Semi-algebraic local-triviality in semi-algebraic mappings, Amer. J. Math. 102 (1980), 291-302.

[Hb] J. H. Hubbard, On the cohomology of Nash sheaves, Topology 11 (1972), 265-270.

[Hr] R. Huber, Isoalgebraische Raüme, Univ. Regensburg, 1984 (preprint).

[Lf] J.-P. Lafon, Séries formelles algébriques, C. R. Acad. Sci. Paris 260 (1965), 3238-3241.

[LaTo] F. Lazzeri and A. Tognoli, Alcune proprietà degli spazi algebrici, Ann. Scuola Norm. Sup. Pisa (3) 24 (1970), 597-632.

[L] S. Łojasiewicz, Ensembles semi-analytiques, I.H.E.S. Bures-sur-Yvette, 1964.

[Mh] L. Mahé, Signatures et composantes connexes, Math. Ann. 260 (1982), 191-210.

[Mo] T. Mostowski, Some properties of the ring of Nash functions, Ann. Scuola Norm. Sup. Pisa Cl. Sci. (4) 3 (1976), 245-266.

[Na] J. Nash, Real algebraic manifolds, Ann. of Math. (2) 56 (1952), 405-421.

[Pe] D. Pecker, On Efroymson's extension theorem for Nash functions, J. Pure Appl. Algebra 37 (1985), 193-203.

[Pu] M. J. de la Puente, The complex spectrum of a ring, Real Algebraic Geometry and Ordered Structures (Baton Rouge, LA, 1996), Contemp. Math., vol. 253, Amer. Math. Soc., Providence, RI, 2000, pp. 235-249.

[Po] D. Popescu, General Néron desingularization, Nagoya Math. J. 100, 104, $118(1985,1986$, 1990), 97-126, 95-115, Letter to the editor: 45-53.

[Qz1] R. Quarez, The Artin conjecture for Q-algebras, Rev. Mat. Univ. Complut. Madrid 10 (1997), 229-263.

[Qz2] _ The idempotency of the real spectrum implies the extension theorem for Nash functions, Math. Z. 227 (1998), 555-570.

[Qz3] _ The Noetherian space of abstract locally Nash sets, Comm. Algebra 26 (1998), 19451966.

[Ra] R. Ramanakoraisina, Complexité des fonctions de Nash, Comm. Algebra 17 (1989), 1395-1406.

[Ri] J.-J. Risler, Sur l'anneau des fonctions de Nash globales, Ann. Sci. École Norm. Sup. (4) 8 (1975), 365-378.

[Rt] C. Rotthaus, On the approximation property of excellent rings, Invent. Math. 88 (1987), 39-63.

[Ry] M.-F. Roy, Faisceau structural sur le spectre réel et fonctions de Nash, Real Algebraic Geometry and Quadratic Forms (Rennes, 1981), Lecture Notes in Math., vol. 959, Springer, Berlin, 1982, pp. 406-432.

[RzSh] J. M. Ruiz and Masahiro Shiota, On global Nash functions, Ann. Sci. École Norm. Sup. (4) 27 (1994), 103-124. 
[Sc] C. Scheiderer, Real and étale cohomology, Lecture Notes in Mathematics, vol. 1588, SpringerVerlag, Berlin, 1994, ISBN 3-540-58436-6.

[Se] A. Seidenberg, Constructions in algebra, Trans. Amer. Math. Soc. 197 (1974), 273-313.

[Sh1] M. Shiota, Nash manifolds, Lecture Notes in Mathematics, vol. 1269, Springer-Verlag, Berlin, 1987, ISBN 3-540-18102-4.

[Sh2] _ Extension et factorisation de fonctions de Nash $C^{\infty}$, C. R. Acad. Sci. Paris Sér. I Math. 308 (1989), 253-256.

[Sh3] _ Geometry of subanalytic and semialgebraic sets, Progress in Mathematics, vol. 150, Birkhäuser Boston Inc., Boston, MA, 1997, ISBN 0-8176-4000-2.

[Sp] M. Spivakovsky, A new proof of D. Popescu's theorem on smoothing of ring homomorphisms, J. Amer. Math. Soc. 12 (1999), 381-444.

[TaTo1] A. Tancredi and A. Tognoli, On the extension of Nash functions, Math. Ann. 288 (1990), 595-604.

[TaTo2] _ On the relative Nash approximation of analytic maps, Rev. Mat. Complut. 11 (1998), 185-200.

[Te] B. Teissier, Résultats récents sur l'approximation des morphismes en algèbre commutative (d'après André, Artin, Popescu et Spivakovsky), Astérisque (1995), Exp. No. 784, 4, 259-282, Séminaire Bourbaki 1993/94.

[To] A. Tognoli, Su una congettura di Nash, Ann. Scuola Norm. Sup. Pisa (3) 27 (1973), 167-185. 